\title{
Enzymatic Synthesis of Ampicillin: Nonlinear Modeling, Kinetics Estimation, and Adaptive Control
}

\author{
Monica Roman and Dan Selişteanu \\ Department of Automatic Control, University of Craiova, A.I. Cuza no. 13, Craiova 200585, Romania \\ Correspondence should be addressed to Dan Selişteanu, dansel@automation.ucv.ro
}

Received 14 October 2011; Accepted 5 December 2011

Academic Editor: T. Akutsu

Copyright (C) 2012 M. Roman and D. Selişteanu. This is an open access article distributed under the Creative Commons Attribution License, which permits unrestricted use, distribution, and reproduction in any medium, provided the original work is properly cited.

\begin{abstract}
Nowadays, the use of advanced control strategies in biotechnology is quite low. A main reason is the lack of quality of the data, and the fact that more sophisticated control strategies must be based on a model of the dynamics of bioprocesses. The nonlinearity of the bioprocesses and the absence of cheap and reliable instrumentation require an enhanced modeling effort and identification strategies for the kinetics. The present work approaches modeling and control strategies for the enzymatic synthesis of ampicillin that is carried out inside a fed-batch bioreactor. First, a nonlinear dynamical model of this bioprocess is obtained by using a novel modeling procedure for biotechnology: the bond graph methodology. Second, a high gain observer is designed for the estimation of the imprecisely known kinetics of the synthesis process. Third, by combining an exact linearizing control law with the online estimation kinetics algorithm, a nonlinear adaptive control law is designed. The case study discussed shows that a nonlinear feedback control strategy applied to the ampicillin synthesis bioprocess can cope with disturbances, noisy measurements, and parametric uncertainties. Numerical simulations performed with MATLAB environment are included in order to test the behavior and the performances of the proposed estimation and control strategies.
\end{abstract}

\section{Introduction}

The design and implementation of modern control strategies in bioindustry require useful models of the biotechnological processes. In many real-life applications, the dynamic models are high-order and nonlinear [1-3]. The bioprocess modeling is a quite difficult task; still, by using the mass balance of the components inside the process and obeying some modeling rules, a dynamical state-space model can be obtained [1-3].

A practical alternative to the classical modeling is the bond graph method, introduced by Paynter in 1961, and further developed in [4]. In the last period, there have been a lot of works on the subject of the theory and application of bond graphs for different kind of systems, such as electrical [5], mechanical, hydraulic, thermal, and chemical [6-8]. This method provides a uniform manner to describe the dynamical behavior for all types of physical systems. The advantages of bond graph modeling are the following: offers a unified approach for all types of systems; allows to display the exchange of power in a system by its graphical representation; due to causality assignment, it gives the possibility of localization of the state variables and achieving the mathematical model in terms of state space equations in an easier way than using classical methods; provides information regarding the structural properties of the system, in terms of controllability observability, and so forth. The bond graph method uses the exchange power in a system, which is normally the product between the effort and flow variables in the true bond graph [5]. Besides this representation there is another one, in which the product effort-flow does not have the physical dimension of power, called pseudo bond graph $[7,8]$. Pseudo bond graphs are more suitable for chemical systems due to the physical meaning of the effort and flow variables. The bond graph modeling of a few biological systems was reported in some works, such as [9]. Though, the bond graph modeling of biotechnological processes is not fully exploited yet; in recent years, only some applications in wastewater treatment bioprocesses were reported $[3,10,11]$. 
To obtain the model of a bioprocess it is necessary to know the fundaments of the bioprocess and to have good knowledge concerning the bond graph methodology. Even if for a bioreactor specialist seems to be easily to obtain the model via classical method, the bond graph approach can be applied without difficulty after a short training. The major advantage of the proposed approach is that the obtained bond graph models of the bioprocesses can be easily used and extended to other bioprocesses. For example, the bond graph models can be used in order to obtain complex models of interconnected bioprocesses [11], and for the direct design of some observers and controllers [12].

The use of modern control techniques for bioprocesses is hampered by the nonlinearity of this kind of processes and the unavailability of several on-line measurements [2]. Serious problems appear in the measurement of biological variables, that is, the substrates, biomass and product concentrations, and so forth. In many cases, the state variables (concentrations) are analyzed manually and as a result there is not on-line (and real-time) control. These various issues can be solved using "software sensors." A software sensor is a combination between a hardware sensor and a software estimator. These software sensors can be used not only for the estimation of the concentrations (state variables), but also for the estimation of the kinetic parameters. Very important is the estimation of kinetic rates inside a bioreactor, that is, the so-called kinetics of the bioprocess. The growing interest for development of software sensors for bioprocess and biological systems is proven by the numerous publications and applications in this field [1$3,13-16]$. One of the first approaches from historically point of view is based on Kalman filter which leads to complex nonlinear algorithms. Another classical technique is the Bastin and Dochain approach based on adaptive systems theory [1]. This strategy consists in the estimation of unmeasured state with asymptotic observers, and after that, the measurements and the estimates of state variables are used for on-line estimation of kinetic rates. This method is useful, but in some cases, when many reactions are involved, the implementation requires the calibration of too many parameters. For instance, if we have $n$ components' concentrations used for the estimation of $m$ kinetic rates, it is necessary to calibrate $2 n$ tuning parameters [1]. In order to overcome this problem, a possibility is to design an estimator using a high gain approach (see $[13,14])$. The gain expression of the high gain observers involves a single tuning parameter whatever the number of components and reactions. High gain observers have evolved over the past two decades as an important tool for the design of output feedback control of nonlinear systems [17]. The early work on high gain observers appeared in the late 1980s, and afterwards the technique was developed independently by two schools of researchers: a French school lead by Gauthier, Hammouri, Farza, and others, $[13,14]$, and a US school lead by Khalil [17].

The modeling techniques and the estimation strategies were used for the design of several control strategies, such as optimal control [1], sliding mode control [18], adaptive control $[1,18]$, vibrational control, model predictive control
[19], fuzzy and neural strategies, and so on. Generally speaking, due to specificity and nonlinearity of bioprocesses, there is no universal solution to the control problem, and good solutions are given only by studying each particular bioprocess.

In this paper, which is an extended work of [20], three main correlated issues concerning the enzymatic bioprocess of ampicillin are studied: modeling, kinetics estimation, and control. First, a pseudo bond graph approach is proposed for the modeling of an enzymatic synthesis of ampicillin, widely used in bioindustry. Ampicillin (6-[2-amino-2-phenylacetamide] penicillanic acid) is a semisynthetic $\beta$-lactamic antibiotic which is very stable at acidic conditions, well absorbed and effective against a wide variety of microorganisms, with low minimal inhibitory concentration [21-24]. Currently, it is manufactured in bioindustry through a chemical route. These reactions typically involve costly steps, such as very low temperatures and the use of toxic organic solvents like methylene chloride and silylation reagents. Enzymatic synthesis is an alternative process; it has high selectivity, specificity, and activity in mild reaction conditions (aqueous medium, neutral $\mathrm{pH}$, and moderate temperatures) [25-27]. The main aim of enzyme immobilization is the industrial reuse of enzymes for many reaction cycles $[25,28,29]$. Thus, simplicity and improvement of enzyme properties have to be strongly associated with the design of protocols for enzyme immobilization. A critical review of enzyme immobilization was presented in [25]. Concerning the use of enzymatic methods in production of semisynthetic $\beta$ lactamic antibiotics, several drawbacks and perspectives are presented by Volpato et al. [30]. These antibacteria agents are produced in hundreds tons/year scale. They are usually produced by the hydrolysis of natural antibiotics (penicillin $G$ or cephalosporin $C$ ). Due to the contaminant reagents used in conventional chemical route, as well as the high energetic consumption, biocatalytic approaches have been studied for both steps in the production of these very interesting medicaments during the last decades [30]. The hydrolysis of penicillin $G$ to produce 6-APA catalyzed by penicillin $G$ acylase is one of the most successful examples of the enzymatic biocatalysis. The dynamical model of this complex bioprocess is obtained via the bond graph methodology by using the reaction scheme and the analysis of biochemical phenomena inside the bioreactor.

Second, because the kinetic rates of the enzymatic bioprocess of ampicillin production are nonlinear and highly uncertain, an on-line estimation strategy is designed. Some estimation strategies were proposed in the last years, such as observer-based estimators and the second-order observers (see, e.g., [31]). In this paper, the design and implementation of high gain observers is proposed, with certain advantages concerning the robustness against disturbances and the simple tuning. The high gain estimation scheme does not require any model for the kinetic rates. The tuning of the proposed observers is reduced to the calibration of a single parameter. The nonlinear observer design is based on the work of Gauthier et al. [14], Farza et al. [13], focused on deriving global results under global Lipschitz conditions. 
Third, an adaptive control law for the enzymatic fedbatch bioprocess of ampicillin production is developed. For bioprocesses taking place into fed-batch reactors, the adaptive control approach is a viable alternative of the optimal control $[1,2]$. In order to design a control law, the so-called exact linearizing approach is used [32]. The nonlinear controller thus obtained is combined with the high gain estimator for the unknown kinetics, and consequently an adaptive controller is obtained. Due to the fact that the implementation of highgain observer and of the adaptive controller requires on-line state estimates, these will be provided by an asymptotic observer. The performances and the behavior of the estimation and control algorithms are studied by using extensive numerical simulations. All these simulations are achieved by using the development, programming and simulation environment Matlab (registered trademark of The MathWorks, Inc., USA).

The results obtained in this study show a good behavior of the adaptive controlled ampicillin synthesis bioprocess. The proposed adaptive control scheme is quite simple, because only two tuning parameters were used, one for estimator and one for the linearizing controller. The bond graph modeling, estimation, and control strategies can be also applied to other processes belonging to the nonlinear class of bioprocesses considered in the study.

\section{Materials and Methods}

2.1. Bond Graph Modeling Method for the Enzymatic Synthesis of Ampicillin. The bioprocesses are highly complex processes that take place inside biochemical reactors (bioreactors). The bioreactors can operate in three modes: the continuous mode, the fed-batch mode, and the batch mode [1-3]. A FedBatch Bioreactor (FBB) initially contains a small amount of substrates (the nutrients) and microorganisms and is progressively filled with the influent substrates. When the FBB is full, the content is harvested. A Batch Bioreactor is filled with the reactant mixture: substrates and microorganisms and allows for a particular time period for the reactions inside the reactor; after some time the products are removed from the tank. In a Continuous Stirred Tank Bioreactor (CSTB), the substrates are fed to the bioreactor continuously and an effluent stream is continuously withdrawn such that the culture volume is constant.

Next, the bond graph method is used in order to obtain the model of the enzymatic synthesis of ampicillin process, which takes place inside an FBB. Firstly, after a short presentation of the bond graph method, a simple prototype fed-batch bioprocess is modeled. After that, the enzymatic synthesis of ampicillin is widely studied, and the bond graph model is obtained by using the reaction scheme, the analysis of the phenomena inside the bioprocess and the bond graph modeling rules.

Bond graph technique uses the effort-flow analogy to describe physical processes. A bond graph consists of subsystems linked together by lines representing power bonds. Each process is described by a pair of variables, effort $e$ and flow $f$, and their product is the power. The direction of power is depicted by a half-arrow. In a dynamic system the effort and the flow variables, and hence the power fluctuate in time. One of the advantages of bond graph method is that models of various systems belonging to different engineering domains can be expressed using a set of only nine elements. A classification of bond graph elements can be made up by the number of ports; ports are places where interactions with other processes take place. There are one port elements represented by inertial elements (I), capacitive elements (C), resistive elements (R), effort sources (Se), and flow sources $(S f)$, two ports elements represented by transformer elements (TF) and gyrator elements (GY), and multiport elements effort junctions (J0), and flow junctions (J1). I, C, and $R$ elements are passive elements because they convert the supplied energy into stored or dissipated energy. Se and Sf elements are active elements because they supply power to the system and TF, GY, 0 -and 1-junctions are junction elements that serve to connect I, C, R, Se, and $S f$, and constitute the junction structure of the bond graph model. Besides the power variables, two other types of variables are very important in describing dynamic systems and these variables, sometimes called energy variables, are the generalized momentum $p$ as time integral of effort and the generalized displacement $q$ as time integral of flow [4].

In biotechnology, pseudo bond graph models are accomplished starting with processes reactions schemes and using both base bond graph elements and pseudo bonds with effort and flow variables as concentrations and mass flows. One of the simplest biological reactions is the microorganisms growth process, with the reaction scheme [1] given by:

$$
S \stackrel{\varphi}{\longrightarrow} X
$$

where $S$ is the substrate, $X$ is the biomass and $\varphi$ is the reaction rate.

This simple growth reaction represents in fact a prototype reaction, which can be found in almost every bioprocess. The dynamics of the concentrations of the components from reaction scheme (1) can be modeled considering the mass balance of the components. The dynamical model of process (1) is simple, but if the reaction scheme is more complicated, the achievement of the dynamical model is difficult. In order to model bioprocesses, pseudo bond graph method is more appropriate because of the meaning of variables involved-effort (concentration) and flow (mass flow). From the reaction scheme (1) and taking into account the mass transfer through the $\mathrm{FBB}$, using the bond graph modeling characteristics, the pseudo bond graph model of the fed-batch bioprocess is achieved see Figure 1. The bond graph model is depicted in the 20 sim environment (registered trademark of Controllab Products B.V. Enschede, The Netherlands).

The directions of half arrows correspond to the run of the reaction, going out from the substrate $S$ towards biomass $X$. In the bond graph model, the mass balances of the species involved in the bioreactor are represented by two 0 junctions: $0_{1,2,3,4}$ (mass balance for the substrate $S$ ) and $0_{7,8,9}$ (mass balance for the biomass $X$ ). Due to the fact that the form of kinetics is complex, nonlinear, and in many cases unknown, the modeling of the reaction kinetics is a difficult 
task. A general assumption [1] is that a reaction can take place only if all reactants are presented in the bioreactor. Therefore, the reaction rates are necessarily zero whenever the concentration of one of the reactants is zero.

In order to model the reaction rate $\varphi$, because of the dependency of substrate and biomass concentrations, we have used a modulated two port $R$ element, denoted $M_{5,6}$. Mass flow of the component entering the reaction is modeled using a modulated source flow element $S f_{1}$ and quantities exiting from the reaction are modeled using modulated flow sources elements $S f$ represented by bonds 3 and 9 . This approach was imposed by the dependency of these elements on $F_{\text {in }}$ : the input feed rate, and on $V$ : volume of the bioreactor.

From $S f$ constitutive equations we have $f_{3}=e_{3} S f_{3}$, $f_{9}=e_{9} S f_{9}$. The accumulations of substrate and biomass in FBB are represented by bonds 2 and 8 and are modeled using capacitive elements $C$, with the constitutive equations:

$$
\begin{gathered}
e_{2}=\frac{q_{2}}{C_{2}}=\frac{\left(\int_{t}\left(f_{1}-f_{3}-f_{4}\right) d t\right)}{C_{2}}, \\
e_{8}=\frac{q_{8}}{C_{8}}=\frac{\left(\int_{t}\left(f_{7}-f_{9}\right) d t\right)}{C_{8}} .
\end{gathered}
$$

By using the constitutive relations of transformer elements $\mathrm{TF}_{4,5}$ and $\mathrm{TF}_{6,7}$, the relations for flows $f_{4}$ and $f_{7}$ are obtained: $f_{4}=k_{4,5} f_{5}, f_{7}=f_{5} / k_{6,7}$, with $k_{4,5}$ and $k_{6,7}$ the transformers modulus, which are in fact yield coefficients of the bioprocess (their values equal one for this fed-batch bioprocess). In fact, $e_{2}$ is the substrate concentration, which will be denoted with $S, e_{8}$ is the biomass concentration $X, f_{5}$ is proportional to $\varphi$ and $V, C_{2}=C_{8}=V$ with $S f_{3}=S f_{9}=$ $F_{\text {in }}$. Therefore, from (2) and (3) we will obtain the dynamical model of the fed-batch bioprocess:

$$
\begin{aligned}
V \frac{d S}{d t} & =V \cdot \dot{S}(t)=F_{\text {in }} S_{\text {in }}-F_{0} S-\varphi V, \\
V \frac{d X}{d t} & =V \cdot \dot{X}(t)=-F_{0} X+\varphi V, \\
\frac{d V}{d t} & =\dot{V}(t)=F_{\text {in }} .
\end{aligned}
$$

The model (4) expresses the equations of mass balance for the reaction scheme (1). Taking into account that the dilution rate $D=F_{\text {in }} / V$, the dynamical behavior of the concentrations can be easily obtained from (4):

$$
\begin{aligned}
& \dot{S}(t)=D S_{\text {in }}-D S-\varphi, \\
& \dot{X}(t)=-D X+\varphi, \\
& \dot{V}(t)=F_{\text {in }} .
\end{aligned}
$$

Next, this bond graph procedure is extended to the modeling of the enzymatic synthesis of ampicillin taking place inside a fed-batch bioprocess. Ampicillin (6-[2-amino2 -phenylacetamide] penicillanic acid) is a semisynthetic $\beta$ lactamic antibiotic which is very stable at acidic conditions,

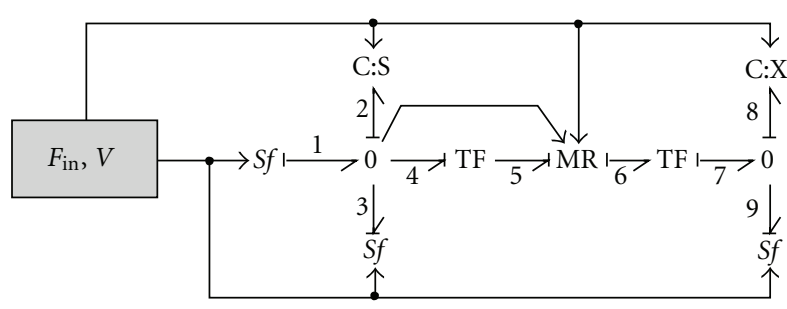

Figure 1: Pseudo bond graph model of the fed-batch prototype bioprocess. The directions of half arrows correspond to the run of the reaction, from the substrate $S$ towards biomass $X$. The mass balances are represented by two 0 -junctions: $0_{1,2,3,4}$ (for $S$ ), and $0_{7,8,9}$ (for $X$ ). Mass flows of entering/exiting components are modeled using modulated source flows $S f$. The reaction rate is modeled by a modulated two port $R$ element, denoted $M_{5,6} . F_{\text {in }}$ is the input feed rate $(l / h)$ and $V$ the bioreactor volume (l).

well absorbed and effective against a wide variety of microorganisms, with low minimal inhibitory concentration [2124]. Currently, it is manufactured in bioindustry through a chemical route. For instance, an amino $\beta$-lactam, such as 6-aminopenicilanic acid (6-APA), having its carboxyl group protected, reacts with an activated side-chain derivative (Dphenylglycine acid chloride, to produce ampicillin). The protecting group is than removed by hydrolysis. These reactions typically involve costly steps, such as very low temperatures and the use of toxic organic solvents like methylene chloride and silylation reagents [22]. Enzymatic synthesis is an alternative process; it has high selectivity, specificity, and activity in mild reaction conditions (aqueous medium, neutral $\mathrm{pH}$ and moderate temperatures). The influence of the substrate structure on the catalytic properties of penicillin $\mathrm{G}$ acylase (PGA) from Escherichia coli in kinetically controlled acylations has been studied in [27]. In particular, the affinity of different $\beta$-lactam nuclei towards the active site has been evaluated considering the ratio between the rate of synthesis and the rate of hydrolysis of the acylating ester. It has been shown that this approach presents several advantages compared with the classical chemical processes [27].

Still, none of the known enzymatic methods have yet been upscaled to industrial applicability, due to the high costs caused by a low yield. Penicillin G acylase (PGA), for example, can act as a hydrolase as well as a transferase, which means that the same enzyme catalyzes the synthesis of ampicillin as well as the hydrolysis of the activated acyl donor and the hydrolysis of the newly formed antibiotic. The main reactions involved in the enzymatic synthesis of ampicillin are presented in Figure $2[33,34]$.

The reaction scheme of this complex bioprocess contains three reactions [31]:

(a) the ampicillin synthesis:

$$
k_{1} S_{1}+S_{2} \stackrel{\varphi_{1}}{\longrightarrow} k_{5} P_{2}+k_{6} P_{3}
$$

(b) the phenylglycine methyl ester hydrolysis:

$$
S_{2} \stackrel{\varphi_{2}}{\longrightarrow} k_{3} P_{1}+k_{7} P_{3}
$$




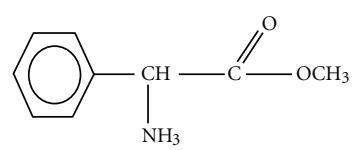

Phenylglycine methyl ester (PGME)

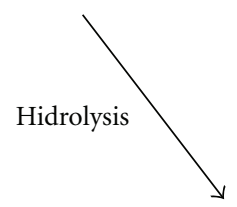

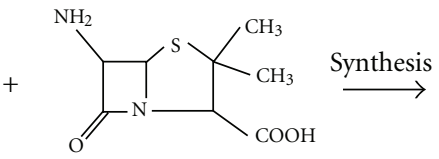

6-aminopenicilanic acid (6-APA)
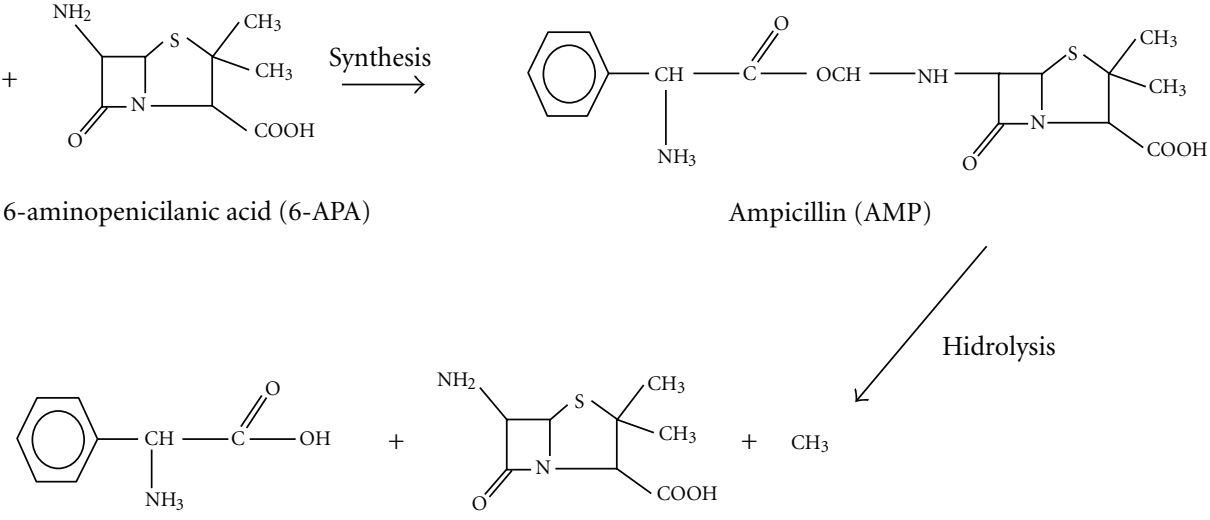

Ampicillin (AMP)

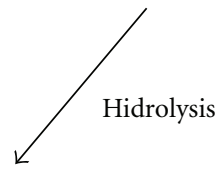

Phenylglycine (PG)

6-aminopenicilanic acid (6-APA)

FIGURE 2: Reactions of enzymatic synthesis of ampicillin [33]. The reaction scheme contains three main reactions: the ampicillin synthesis, the phenylglycine methyl ester hydrolysis, and the ampicillin hydrolysis.

(c) the ampicillin hydrolysis:

$$
P_{2} \stackrel{\varphi_{3}}{\longrightarrow} k_{4} P_{1}+k_{2} S_{1}
$$

In the above reactions, $S_{1}, S_{2}, P_{1}, P_{2}$, and $P_{3}$ represent 6-aminopenicillanic acid (6-APA), phenylglycine methyl ester (PGME), phenylglycine (PG), ampicillin (AMP), and methanol, respectively. $\varphi_{1}, \varphi_{2}$, and $\varphi_{3}$ represent the reaction rates of these three reactions, and $k_{i}$ are yield coefficients. Considering that the bioprocess takes place inside a fed-batch bioreactor, from the reaction scheme (6)-(8), and by using the bond graph elements and modeling rules, a bond graph model of the process is obtained see Figure 3.

As it is mentioned previously, the meanings of the effort and flow variables of the bond graph model are concentration and mass flow, respectively. The directions of the half-arrows in the bond graph correspond to the progress of the reactions, going out from the components $S_{1}$ and $S_{2}$ towards $P_{2}$ and $P_{3}$ for the first reaction, from $S_{2}$ to $P_{1}$ and $P_{3}$ for the second reaction, and from $P_{2}$ towards $P_{1}$ and $S_{1}$ for the third reaction, respectively. In bond graph terms, the mass balances of the components involved in the bioprocess are represented by five 0 -junctions: $0_{1,2,3,4,33}$ (mass balance for $S_{1}$ ), $0_{6,7,8,9,11}$ (mass balance for $S_{2}$ ), $0_{15,16,17,18,31}$ (mass balance for $P_{1}$ ), $0_{20,21,22,27}$ (mass balance for $P_{2}$ ), and $0_{24,25,26,36}$ (mass balance for $P_{3}$ ). The constitutive relations of these junctions are characterized by the equality to zero of the sum of flow variables corresponding to the junction bonds; therefore, the next relations are obtained:

$$
\begin{gathered}
f_{1}-f_{2}-f_{3}-f_{4}+f_{33}=0, \quad f_{6}-f_{7}-f_{8}-f_{9}-f_{11}=0 \\
f_{15}-f_{16}-f_{17}+f_{18}+f_{31}=0, \quad f_{20}-f_{21}-f_{22}-f_{27}=0 \\
f_{24}-f_{25}-f_{26}+f_{36}=0 .
\end{gathered}
$$

The accumulations of components $S_{1}, S_{2}, P_{1}, P_{2}$, and $P_{3}$ in the bioreactor are represented by bonds $2,7,16,22$, and
25, and they are modeled using capacitive elements $C$. The constitutive equations of $C$-elements are as follows:

$$
\begin{aligned}
& e_{2}=\frac{1}{C_{2}} q_{2}=\frac{1}{C_{2}} \int_{t}\left(f_{1}-f_{3}-f_{4}+f_{33}\right) d t, \\
& e_{7}=\frac{1}{C_{7}} q_{7}=\frac{1}{C_{7}} \int_{t}\left(f_{6}-f_{8}-f_{9}-f_{11}\right) d t, \\
& e_{16}=\frac{1}{C_{16}} q_{16}=\frac{1}{C_{16}} \int_{t}\left(f_{15}-f_{17}+f_{18}+f_{31}\right) d t, \\
& e_{22}=\frac{1}{C_{22}} q_{22}=\frac{1}{C_{22}} \int_{t}\left(f_{20}-f_{21}-f_{27}\right) d t, \\
& e_{25}=\frac{1}{C_{25}} q_{25}=\frac{1}{C_{25}} \int_{t}\left(f_{24}-f_{26}+f_{36}\right) d t,
\end{aligned}
$$

where $e_{2}, e_{7}, e_{16}, e_{22}, e_{25}$ are the concentrations of components $S_{1}, S_{2}, P_{1}, P_{2}, P_{3}$ (all in $\mathrm{mM}=10^{-3} \mathrm{~mol} / \mathrm{L}$ ), and $C_{2}, C_{7}, C_{16}, C_{22}, C_{25}$ are the parameters of $C$-elements: $C_{2}=$ $C_{7}=C_{16}=C_{22}=C_{25}=V$, with $V$ the bioreactor volume (L).

The output flows of the components exiting from the reactions are modeled by using flow source elements $\mathrm{Sf}$ represented by bonds $3,8,17,21$, and 26 . The constitutive equations of these elements are as follows: $f_{3}=$ $S f_{3}, f_{8}=S f_{8}, f_{17}=S f_{17}, f_{21}=S f_{21}, f_{26}=S f_{26}$, where $S f_{3}, S f_{8}, S f_{17}, S f_{21}, S f_{26}$ are the parameters of Sf-elements. Therefore we have $S f_{3}=S f_{8}=S f_{17}=S f_{21}=S f_{26}=$ $F_{0}$, where $F_{0}$ is the output flow $(\mathrm{L} / \mathrm{min})$. Mass flows of the components entering the reactions are modeled using source flow elements $S f_{1}, S f_{6}$, and $S f_{18}$. Consequently, we have $f_{1}=V F_{1}, f_{6}=V F_{2}, f_{18}=V F_{3}$, where $F_{1}, F_{2}$ and $F_{3}$ (all in $\mathrm{mM} / \mathrm{min}$ ) are the feed rates of 6 -APA, PGME, and PG, respectively. The transformer elements $\mathrm{TF}_{4,5}, \mathrm{TF}_{9,10}, \mathrm{TF}_{11,12}$, $\mathrm{TF}_{14,15}, \mathrm{TF}_{19,20}, \mathrm{TF}_{23,24}, \mathrm{TF}_{27,28}, \mathrm{TF}_{30,31}, \mathrm{TF}_{32,33}, \mathrm{TF}_{35,36}$ were introduced to model the yield coefficients. For the modeling of the reaction rates we used three two-port modulated $R$ elements: MR1, MR2, and MR3. From the constitutive relations of the three 1 -junction elements, $1_{5,10,19,34}, 1_{12,13,23}$, 


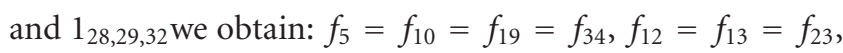
$f_{28}=f_{29}=f_{32}$, where the constitutive relations of MR elements imply that $f_{34}=\varphi_{1} V, f_{13}=\varphi_{2} V$, and $f_{29}=$ $\varphi_{3} V$. Also, from the constitutive relations of transformers we obtain the following relations:

$$
\begin{aligned}
& f_{4}=k_{4,5} f_{5}, \quad f_{9}=k_{9,10} f_{10}, \quad f_{11}=k_{11,12} f_{12}, \\
& f_{15}=\frac{1}{k_{14,15}} f_{14}, \quad f_{20}=\frac{1}{k_{19,20}} f_{19}, \\
& f_{24}=\frac{1}{k_{23,24}} f_{23}, \quad f_{27}=k_{27,28} f_{28}, \quad f_{31}=\frac{1}{k_{30,31}} f_{30}, \\
& f_{33}=\frac{1}{k_{32,33}} f_{33}, \quad f_{36}=\frac{1}{k_{35,36}} f_{36},
\end{aligned}
$$

with $k_{4,5}, k_{9,10}, k_{11,12}, k_{14,15}, k_{19,20}, k_{23,24}, k_{27,28}, k_{30,31}, k_{32,33}$, and $k_{35,36}$ the transformers modulus, which are in fact yield coefficients of the bioprocess.

Using these notations, from (10)-(14) and the above relationships, the following dynamical model of the bioprocess is obtained:

$$
\begin{aligned}
& V \cdot \dot{S}_{1}=V F_{1}-F_{0} S_{1}-k_{4,5} \varphi_{1} V+\left(\frac{1}{k_{32,33}}\right) \varphi_{3} V, \\
& V \cdot \dot{S}_{2}=V F_{2}-F_{0} S_{2}-k_{9,10} \varphi_{1} V-k_{11,12} \varphi_{2} V, \\
& V \cdot \dot{P}_{1}=\left(\frac{1}{k_{14,15}}\right) \varphi_{2} V-F_{0} P_{1}+V F_{3}+\left(\frac{1}{k_{30,31}}\right) \varphi_{3} V, \\
& V \cdot \dot{P}_{2}=\left(\frac{1}{k_{19,20}}\right) \varphi_{1} V-F_{0} P_{2}-k_{27,28} \varphi_{3} V, \\
& V \cdot \dot{P}_{3}=\left(\frac{1}{k_{23,24}}\right) \varphi_{2} V-F_{0} P_{3}+\left(\frac{1}{k_{35,36}}\right) \varphi_{1} V .
\end{aligned}
$$

Taking into account that $k_{4,5}=k_{1}, k_{32,33}=1 / k_{2}, k_{9,10}=$ $k_{11,12}=1, k_{14,15}=1 / k_{3}, k_{30,31}=1 / k_{4}, k_{19,20}=1 / k_{5}, k_{27,28}=$ $1, k_{35,36}=1 / k_{6}, k_{23,24}=1 / k_{7}$, and using the so-called dilution rate $D=F_{0} / V=1 / t_{r}$, with $t_{r}$ : medium residence time, the equations (16) can be written in the next form:

$$
\begin{aligned}
& \dot{S}_{1}=-k_{1} \varphi_{1}+k_{2} \varphi_{3}-D S_{1}+F_{1}, \\
& \dot{S}_{2}=-\varphi_{1}-\varphi_{2}-D S_{2}+F_{2}, \\
& \dot{P}_{1}=k_{3} \varphi_{2}+k_{4} \varphi_{3}-D P_{1}+F_{3}, \\
& \dot{P}_{2}=k_{5} \varphi_{1}-\varphi_{3}-D P_{2}, \\
& \dot{P}_{3}=k_{6} \varphi_{2}+k_{7} \varphi_{1}-D P_{3} .
\end{aligned}
$$

The model of this bioprocess, obtained via bond graph approach, is equivalent with the model obtained via classical method (see e.g., [31]). The model (17) can be expressed in the form:

$$
\frac{d}{d t}\left[\begin{array}{c}
S_{1} \\
S_{2} \\
P_{1} \\
P_{2} \\
P_{3}
\end{array}\right]=\underbrace{\left[\begin{array}{ccc}
-k_{1} & 0 & k_{2} \\
-1 & -1 & 0 \\
0 & k_{3} & k_{4} \\
k_{5} & 0 & -1 \\
k_{6} & k_{7} & 0
\end{array}\right]}_{K}\left[\begin{array}{l}
\varphi_{1} \\
\varphi_{2} \\
\varphi_{3}
\end{array}\right]-D\left[\begin{array}{c}
S_{1} \\
S_{2} \\
P_{1} \\
P_{2} \\
P_{3}
\end{array}\right]+\left[\begin{array}{c}
F_{1} \\
F_{2} \\
F_{3} \\
0 \\
0
\end{array}\right] .
$$

The state vector of the system (18) will be denoted as: $\xi=\left[\begin{array}{lllll}S_{1} & S_{2} & P_{1} & P_{2} & P_{3}\end{array}\right]^{T}$. The vector of reaction rates is

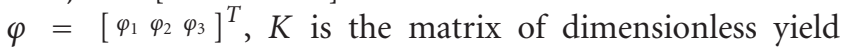
coefficients, and the vector of feed rates is denoted with $F=\left[\begin{array}{llll}F_{1} & F_{2} & F_{3} & 0\end{array}\right]^{T}$. By using these notations, the dynamical nonlinear model (18) can be compactly written as:

$$
\dot{\xi}=K \varphi(\xi)-D \xi+F .
$$

The dynamical model of the enzymatic synthesis of ampicillin (19) belongs to a large class of nonlinear bioprocesses carried out in bioreactors and is referred as general dynamical state-space model of this class of bioprocesses [1].

2.2. On-Line Estimation of Unknown Kinetics with High Gain Observers. The most difficult task for the construction of the dynamical model (19) is the modeling of the reaction rates. The form of kinetics is complex, nonlinear, and in many cases partial or completely unknown. A realistic assumption is that a reaction can take place only if all reactants are presented in the reactor [1]. Thus, the reaction rates are necessarily zero whenever the concentration of one of reactants is zero. Taking into consideration these aspects, the reaction rates can be written as follows:

$$
\varphi(\xi)=\left[\begin{array}{l}
\varphi_{1} \\
\varphi_{2} \\
\varphi_{3}
\end{array}\right]=\underbrace{\left[\begin{array}{ccc}
S_{1} & 0 & 0 \\
0 & S_{2} & 0 \\
0 & 0 & P_{2}
\end{array}\right]}_{H(\xi)}\left[\begin{array}{l}
\mu_{1} \\
\mu_{2} \\
\mu_{3}
\end{array}\right]=H(\xi) \mu(\xi),
$$

where $\mu(\cdot)$ is the vector of specific reaction rates. A possible structure of the nonlinear specific growth rates is of Monodtype model, and it is given in $[22,23,34]$. Consequently, the reaction rates can be modeled as follows:

(a) for the ampicillin synthesis:

$$
\begin{aligned}
\varphi_{1}(\xi) & =S_{1} \mu_{1}(\xi) \\
& =\frac{S_{1}}{k_{\mathrm{EN}}+S_{1}} \cdot \frac{k_{\mathrm{cat} 1} C_{\mathrm{EZ}} S_{2}}{K_{m 1}\left(1+P_{2} / k_{\mathrm{AE}}\right)+S_{2}}
\end{aligned}
$$

(b) for the phenylglycine methyl ester hydrolysis:

$$
\varphi_{2}(\xi)=S_{2} \mu_{2}(\xi)=\frac{k_{\mathrm{cat} 1} C_{\mathrm{EZ}} S_{2}}{K_{m 1}\left(1+P_{2} / k_{\mathrm{AE}}\right)+S_{2}}
$$




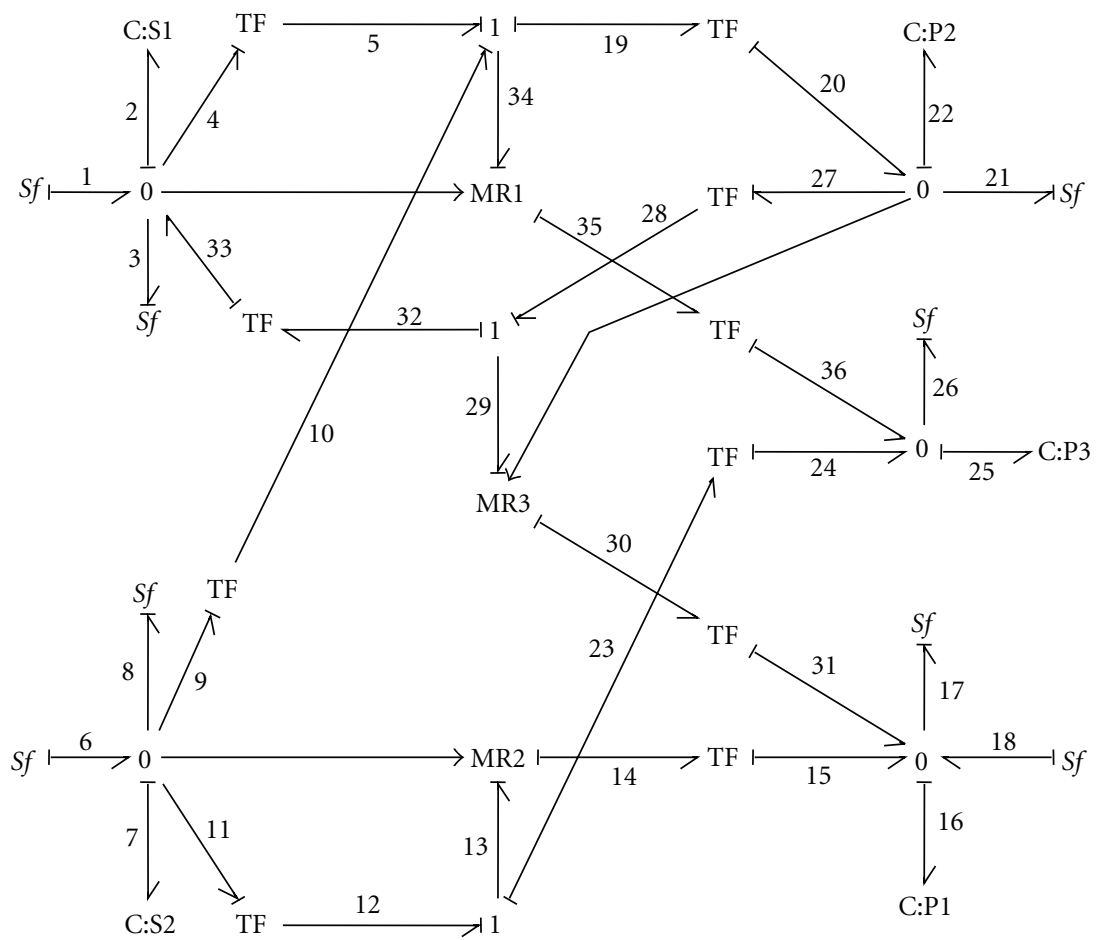

FIGURE 3: Bond graph model of the enzymatic synthesis of ampicillin. The directions of half arrows correspond to the run of the reaction, from the reactants towards the reaction products. The mass balances are represented by five 0 -junctions, and the mass flows of entering/exiting components are modeled using modulated source flows $S f$. The reaction rates are modeled by three modulated two port $\mathrm{R}$ elements, MR1, MR2, and MR3. In order to simplify the model representation, the feed flow and the volume are not shown. The bond graph model was depicted in 20 sim environment.

(c) for the ampicillin hydrolysis:

$$
\varphi_{3}(\xi)=P_{2} \mu_{3}(\xi)=\frac{k_{\mathrm{cat} 2} C_{\mathrm{EZ}} P_{2}}{K_{m 2}\left(1+S_{2} / k_{\mathrm{EA}}\right)+P_{2}},
$$

where $C_{\mathrm{EZ}}$ represents the enzyme activity (UI/mlgel), $k_{\mathrm{EN}}$, $k_{\mathrm{AE}}$, and $k_{\mathrm{EA}}$ are inhibition constants $(\mathrm{mM}), k_{\text {catl }}$, and $k_{\text {cat2 }}$ are kinetic constants (mM/UI min), $K_{m 1}$, and $K_{m 2}$ are Michaelis-Menten constants (mM).

However, in practice the reaction rates and/or the specific reaction rates given by the relations (21)-(23) are imprecisely known. For on-line estimation of these kinetic rates, high gain observers can be designed. Next, the nonlinear model (19) is expressed as:

$$
\dot{\xi}=K H(\xi) \rho(t)-D \xi+F,
$$

where $\rho(t)$ represents the unknown kinetics of the process. If we suppose that the reaction rates are totally unknown, then $\rho(t)=\varphi(t)$ and $H(\xi)=1$. If the structure of the reaction rates is known $\varphi(\xi)=H(\xi) \mu(\xi)$, but the specific reaction rates are unknown, then $\rho(t)=\mu(t)$ and $H(\xi)$ is the matrix given in (20).

The high gain observers design necessitates a factorization of the model $(24)[13,14,20]$. We will consider that the yield matrix $K$ is of full rank, which is true for our particular model, and for general class of bioprocesses' models is a generic property. We shall suppose that all state variables are measured (contrarily, a state estimator can be used). Since the yield matrix $K$ is of full rank, then the partition $\left(K_{a}, K_{b}\right)$ can be considered, such that the submatrix $K_{a}$ has full rank. Therefore, a partition $\left(\xi_{a}, \xi_{b}\right)$ of the state vector is obtained, and consequently a partition for $F$ is achieved: $\left(F_{a}, F_{b}\right)$. Then, the system (24) can be written as follows:

$$
\begin{aligned}
& \dot{\xi}_{a}=K_{a} \cdot H\left(\xi_{a}, \xi_{b}\right) \cdot \rho(t)-D \cdot \xi_{a}+F_{a}, \\
& \dot{\xi}_{b}=K_{b} \cdot H\left(\xi_{a}, \xi_{b}\right) \cdot \rho(t)-D \cdot \xi_{b}+F_{b},
\end{aligned}
$$

By using this factorization, a highgain observer can be implemented. The design of highgain observers is done in $[13,14]$, with supplementary assumptions regarding global Lipschitz conditions, the boundedness of $H(\xi)$ diagonal elements' away from zero, and so forth. The equations of the nonlinear high gain observer for (24) are obtained as [14]:

$$
\begin{aligned}
& \dot{\hat{\xi}}_{a}=K_{a} H\left(\xi_{a}, \xi_{b}\right) \hat{\rho}-D \hat{\xi}_{a}+F_{a}-2 \theta\left(\hat{\xi}_{a}-\xi_{a}\right), \\
& \dot{\hat{\rho}}=-\theta^{2} \cdot\left[K_{a} \cdot \mathrm{H}\left(\hat{\xi}_{a}, \xi_{b}\right)\right]^{-1} \cdot\left(\hat{\xi}_{a}-\xi_{a}\right) .
\end{aligned}
$$

The high gain observer (26) provides on-line estimates $\hat{\rho}$ for the unknown kinetics. This on-line estimation algorithm is in fact a copy of the process model, with a corrective term. The observer is simple and the tuning of the gain can be done by modifying only one design parameter: $\theta$. It should be noticed that $\hat{\xi}_{a}$ is an "estimate" of $\xi_{a}$, provided by the 
algorithm in order to be compared with the real state ( $\xi_{a}$ is measured or provided by a state observer), and the resulting error to be used in (26).

In order to obtain the equations of the observers for our bioprocess, the next factorization of yield matrix and corresponding partition are considered [20]:

$$
\begin{aligned}
& K_{a}=\left[\begin{array}{ccc}
-k_{1} & 0 & k_{2} \\
-1 & -1 & 0 \\
0 & k_{3} & k_{4}
\end{array}\right], \quad K_{b}=\left[\begin{array}{ccc}
k_{5} & 0 & -1 \\
k_{6} & k_{7} & 0
\end{array}\right], \\
& \xi_{a}=\left[\begin{array}{l}
S_{1} \\
S_{2} \\
P_{1}
\end{array}\right], \quad \xi_{b}=\left[\begin{array}{l}
P_{2} \\
P_{3}
\end{array}\right], \\
& F_{a}=\left[\begin{array}{l}
F_{1} \\
F_{2} \\
F_{3}
\end{array}\right], \quad F_{b}=\left[\begin{array}{l}
0 \\
0
\end{array}\right] .
\end{aligned}
$$

Then, for the enzymatic synthesis of ampicillin, two high-gain estimators can be derived from (26) [20].

(a) an estimator for the specific reaction rates. In this case $\rho(t)=\mu(t)$, and $H(\xi)$ is the matrix given in (20). The equations of the high-gain observer are:

$$
\begin{aligned}
{\left[\begin{array}{c}
\dot{\hat{S}}_{1} \\
\hat{\hat{S}}_{1} \\
\hat{\hat{P}}_{1}
\end{array}\right]=} & K_{a}\left[\begin{array}{ccc}
\hat{S}_{1} & 0 & 0 \\
0 & \hat{S}_{2} & 0 \\
0 & 0 & P_{2}
\end{array}\right]\left[\begin{array}{l}
\hat{\mu}_{1} \\
\hat{\mu}_{2} \\
\hat{\mu}_{3}
\end{array}\right]-D\left[\begin{array}{c}
\hat{S}_{1} \\
\hat{S}_{2} \\
\hat{P}_{1}
\end{array}\right] \\
& +\left[\begin{array}{c}
F_{1} \\
F_{2} \\
F_{3}
\end{array}\right]-2 \theta\left[\begin{array}{l}
\hat{S}_{1}-S_{1} \\
\hat{S}_{2}-S_{2} \\
\hat{P}_{1}-P_{1}
\end{array}\right] \\
{\left[\begin{array}{c}
\dot{\hat{\mu}}_{1} \\
\dot{\hat{\mu}}_{2} \\
\dot{\hat{\mu}}_{3}
\end{array}\right]=} & -\theta^{2} \cdot\left[K_{a} \cdot\left[\begin{array}{ccc}
\hat{S}_{1} & 0 & 0 \\
0 & \hat{S}_{2} & 0 \\
0 & 0 & P_{2}
\end{array}\right]\right]^{-1} \cdot\left[\begin{array}{l}
\hat{S}_{1}-S_{1} \\
\hat{S}_{2}-S_{2} \\
\hat{P}_{1}-P_{1}
\end{array}\right],
\end{aligned}
$$

(b) a second estimator can be obtained if the entire reaction rate vector is considered unknown. In this case $\rho(t)=\varphi(t)$ and $H(\xi)=1$. Then, the equations of the highgain observer are as follows:

$$
\begin{aligned}
& {\left[\begin{array}{l}
\dot{\hat{S}}_{1} \\
\dot{\hat{S}}_{1} \\
\dot{\hat{P}}_{1}
\end{array}\right]=K_{a}\left[\begin{array}{l}
\hat{\varphi}_{1} \\
\hat{\varphi}_{2} \\
\hat{\varphi}_{3}
\end{array}\right]-D\left[\begin{array}{l}
\hat{S}_{1} \\
\hat{S}_{2} \\
\hat{P}_{1}
\end{array}\right]+\left[\begin{array}{c}
F_{1} \\
F_{2} \\
F_{3}
\end{array}\right]-2 \theta\left[\begin{array}{l}
\hat{S}_{1}-S_{1} \\
\hat{S}_{2}-S_{2} \\
\hat{P}_{1}-P_{1}
\end{array}\right],} \\
& {\left[\begin{array}{c}
\dot{\hat{\varphi}}_{1} \\
\dot{\hat{\varphi}}_{2} \\
\dot{\hat{\varphi}}_{3}
\end{array}\right]=-\theta^{2} \cdot\left[K_{a}\right]^{-1} \cdot\left[\begin{array}{l}
\hat{S}_{1}-S_{1} \\
\hat{S}_{2}-S_{2} \\
\hat{P}_{1}-P_{1}
\end{array}\right] .}
\end{aligned}
$$

The high gain observer (28) needs the on-line measurements of $S_{1}, S_{2}, P_{1}$, and $P_{2}$. Usually, only the first three concentrations are available. Therefore, a state observer is required in order to reconstitute the measurements of $P_{2}$. For example, an asymptotic state observer is designed in [31]. In such a case, the estimates of $P_{2}$ provided by the asymptotic observer will be used in (28). In the case of the high gain observer (29), only the measurements of $S_{1}, S_{2}$, and $P_{1}$ are needed.

2.3. Adaptive Control Law Design. Concerning the fed-batch bioprocess control, a typical problem is that of generating the substrate feed rate profile to optimize a performance criterion [1]. For our process, the main objective is to obtain a high level of the ampicillin concentration. This goal can be achieved through an optimal control, that is, the calculation of a feeding rate optimal profile. This is unsatisfactory when the kinetics is imprecisely known. A possible suboptimal alternative is the adaptive control [1]. In this section, firstly, an exact linearizing control law is obtained. Then, adaptive versions are implemented, considering that the kinetics are unknown, and by using the kinetics estimators described before.

The exact linearizing control law for the model (18) (or the model written in the compact form (19)) is obtained in a classical three steps strategy (see [32] for the general point of view and [1] for bioprocesses). The control goal is that the ampicillin concentration $y(t)=\xi_{4}(t)=P_{2}(t)$ to track the desired substrate trajectory $y^{*}(t)=P_{2}^{*}(t)$, with the dilution rate as control action: $u(t)=D(t)$. The first step consists in the achievement of an input-output model of the bioprocess, which is obtained directly from (18):

$$
\dot{y}=\dot{P}_{2}=\dot{\xi}_{4}=k_{5} \varphi_{1}(\xi)-\varphi_{3}(\xi)-u \cdot y .
$$

Second, we consider a stable and linear reference model for the tracking error $y^{*}-y$ :

$$
\left(\dot{y}^{*}-\dot{y}\right)+\lambda\left(y^{*}-y\right)=0, \quad \lambda>0 .
$$

Finally, the exact linearizing control law is obtained by calculus of usuch that (30) has the same behavior as (31):

$$
\begin{aligned}
u(t)= & \left(\frac{1}{y}\right) \cdot\left(k_{5} \varphi_{1}(\xi)-\varphi_{3}(\xi)-\lambda\left(y^{*}-y\right)\right) \\
= & \left(\frac{1}{y}\right) \cdot\left(k_{5} \mu_{1}(\xi) \xi_{1}-\mu_{3}(\xi) y-\lambda\left(y^{*}-y\right)\right) \\
= & \left(\frac{1}{y}\right) \cdot\left(k_{5} \frac{\xi_{1}}{k_{\mathrm{EN}}+\xi_{1}} \cdot \frac{k_{\mathrm{cat} 1} C_{\mathrm{EZ}} \xi_{2}}{K_{m 1}\left(1+y / k_{\mathrm{AE}}\right)+\xi_{2}}\right. \\
& \left.\quad-\frac{k_{\mathrm{cat} 2} C_{\mathrm{EZ}} y}{K_{m 2}\left(1+\xi_{2} / k_{\mathrm{EA}}\right)+y}-\lambda\left(y^{*}-y\right)\right),
\end{aligned}
$$

where we consider $y^{*}=$ const.

The exact linearizing control (32) can ensure the achievement of control goal only if the involved concentrations are on-line measurable and if the reaction rates (or the specific growth rates, resp.) are known. Contrarily, the estimations 


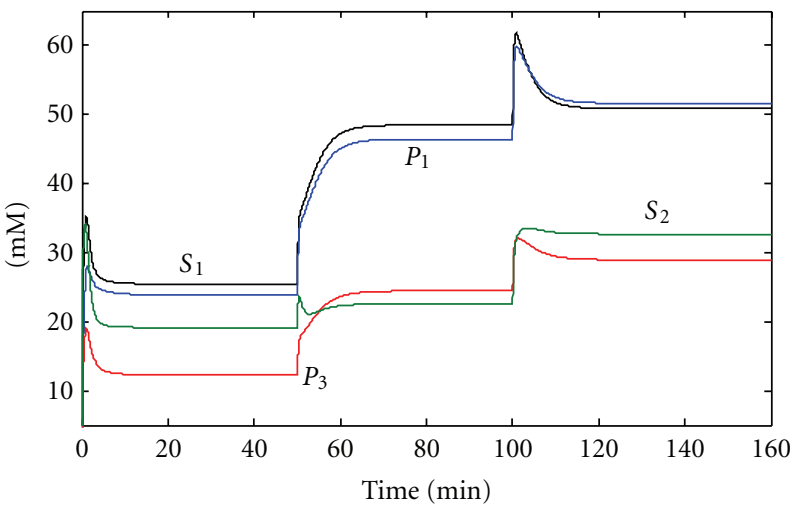

(a)

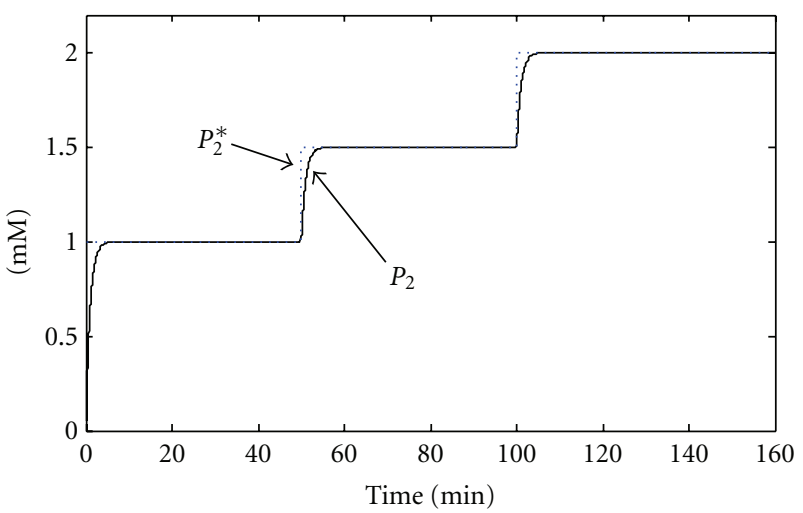

(c)
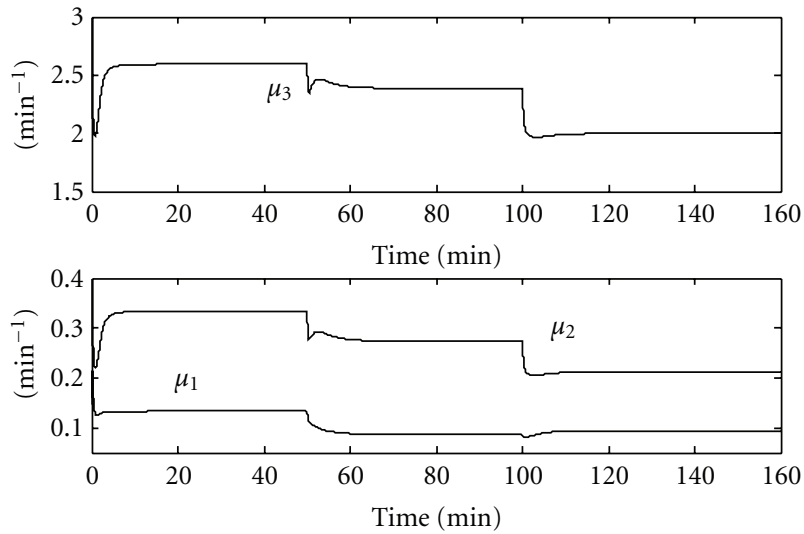

(b)

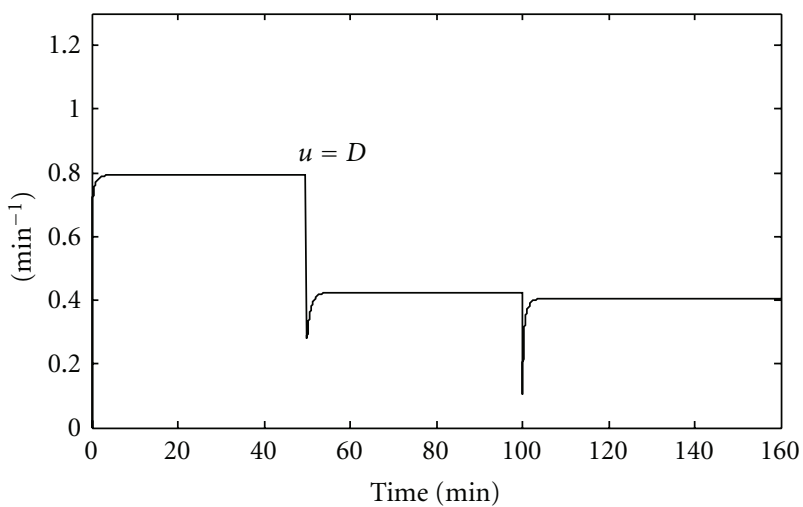

(d)

FIGURE 4: Simulation results-exact linearizing control law. The closed loop system was tested for a step profile of the ampicillin concentration reference. Panel (a) shows the time evolution of $\xi_{1}=S_{1}, \xi_{2}=S_{2}, \xi_{3}=P_{1}$, and $\xi_{5}=P_{3}$ (i.e., 6-APA, PGME, PG, and methanol concentrations, resp.). In panel (b), the profiles of specific reaction rates $\mu_{1}, \mu_{2}$, and $\mu_{3}$ are depicted. Panel (c) presents the output $y=P_{2}$ (ampicillin concentration) versus the reference profile $y^{*}=P_{2}^{*}$. Panel (d) shows the control action, that is, the evolution of the dilution rate.

provided by the estimators are needed, and if they are used in the exact linearizing control law, some adaptive versions of the nonlinear law are obtained. For example, if the estimations $\hat{\rho}(t)=\hat{\mu}(t)$ provided by (28) are used in the control law (32), an adaptive version of this law is obtained as follows:

$$
u(t)=\left(\frac{1}{y}\right) \cdot\left(k_{5} \hat{\mu}_{1}(t) \xi_{1}-\hat{\mu}_{3}(t) y-\lambda\left(y^{*}-y\right)\right)
$$

The entire adaptive control algorithm consists of (28) and (33). Regarding the stability and convergence properties of the controlled system, these are widely discussed for a general class of bioprocesses in [1]. Another version of the adaptive control law (33) is obtained if the full vector of reaction rates is considered as unknown. In this case, the high gain estimator (29) is used, and the adaptive control law takes the following form:

$$
u(t)=\left(\frac{1}{y}\right) \cdot\left(k_{5} \hat{\varphi}_{1}(t)-\hat{\varphi}_{3}(t)-\lambda\left(y^{*}-y\right)\right)
$$

Therefore, the complete adaptive control algorithm consists now of (29) and (34). Moreover, when the ampicillin concentration cannot be on-line measured, then an asymptotic observer [31] can be used in order to provide the estimates $y_{\text {est }}$, and consequently a version of the adaptive controller (33) is obtained as follows:

$$
u(t)=\left(\frac{1}{y_{\mathrm{est}}}\right) \cdot\left(k_{5} \hat{\mu}_{1}(t) \xi_{1}-\hat{\mu}_{3}(t) y_{\mathrm{est}}-\lambda\left(y^{*}-y_{\mathrm{est}}\right)\right) .
$$

The adaptive control algorithm consists of (28), (35), plus the asymptotic observer.

The design of asymptotic observers is based on mass and energy balances without the knowledge of the process kinetics being necessary [1]. More precisely, the design is based on some useful changes of coordinates, which lead to a submodel of the process which is independent of the kinetics. Next, the fundaments of the design of an asymptotic observer for the process (24) will be presented. The maximum state information which can be reconstituted is obtained by using the states $\xi_{1}=\left[\begin{array}{ll}S_{1} & P_{1}\end{array}\right]^{T}$ considered as measurable. Therefore, the vector of unavailable states is $\xi_{2}=\left[\begin{array}{lll}S_{2} & P_{2} & P_{3}\end{array}\right]^{T}$; these 


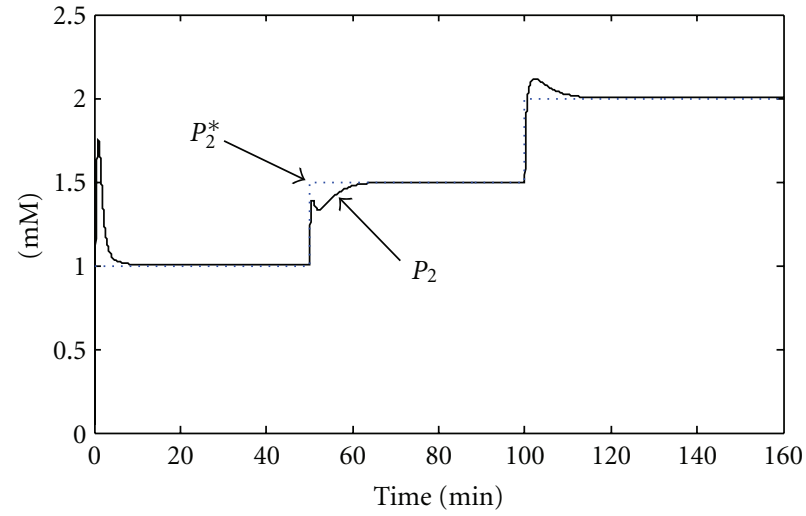

(a)

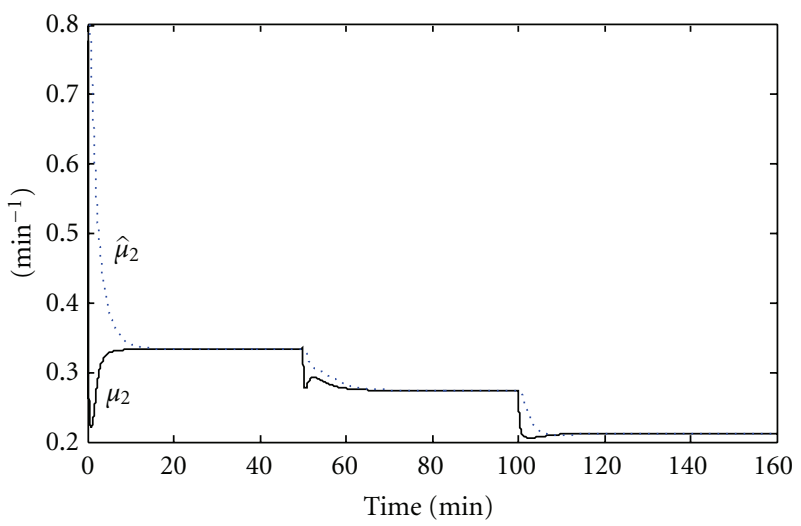

(c)

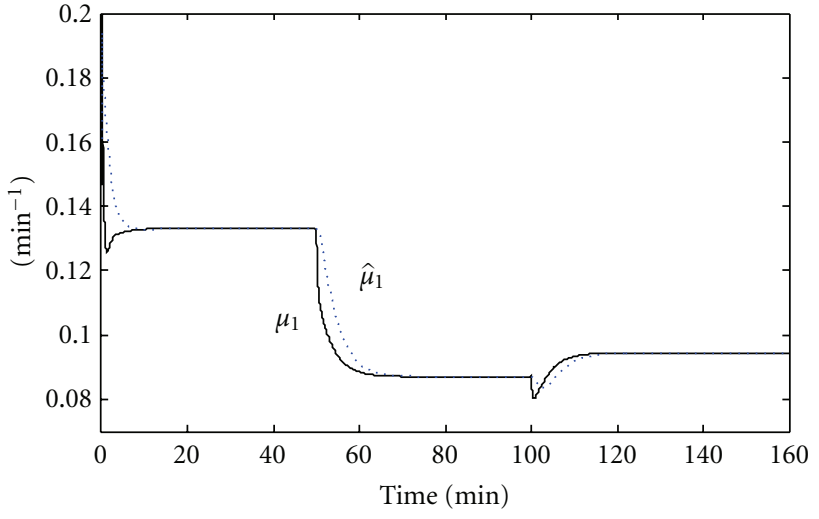

(b)

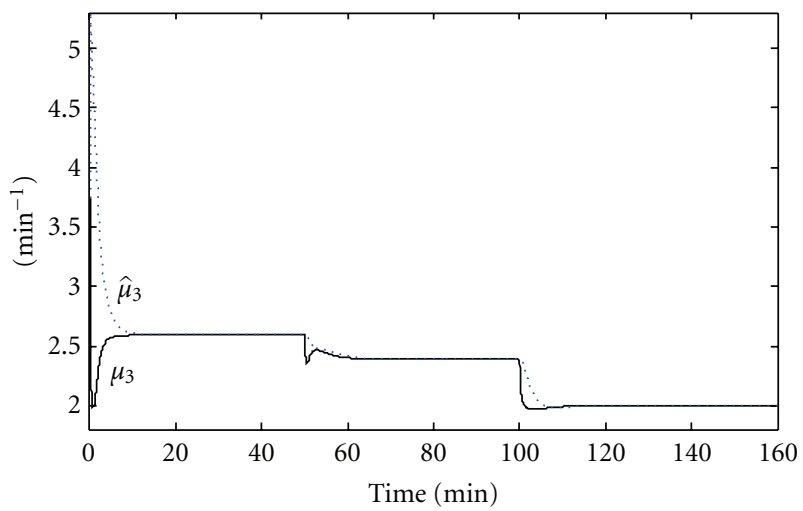

(d)

FIgURE 5: Simulation results-adaptive control law (free-noise measurements). Panel (a) shows the time evolution of the output $y=P_{2}$ (ampicillinconcentration) versus the reference profile $y^{*}=P_{2}^{*}$, in the adaptive case. Panels (b), (c), and (d) present the profiles of specific reaction rates $\mu_{1}, \mu_{2}$, and $\mu_{3}$ together with their estimates $\hat{\mu}_{1}, \hat{\mu}_{2}$, and $\hat{\mu}_{3}$, respectively, estimates provided by the nonlinear observer.

states will be estimated. In order to achieve the change of coordinates, the partition $\left(\xi_{1}, \xi_{2}\right)$ induces partitions of the yield matrix $K:\left(K_{1}, K_{2}\right)$, also of the rate vector $\left(\widetilde{F}_{1}, \widetilde{F}_{2}\right)$ accordingly. The state partition was chosen such that the submatrix $K_{1}$ is full rank and $\operatorname{dim}\left(\xi_{1}\right)=\operatorname{rank}\left(K_{1}\right)=$ $\operatorname{rank}(K)$. Then a linear change of coordinates can be defined as follows:

$$
z=G \cdot \xi_{1}+\xi_{2}
$$

with $z$ an auxiliary state vector and $\mathrm{G}$ the solution of the matrix equation $G \cdot K_{1}+K_{2}=0$. In the new coordinates, model (19) can be rewritten as

$$
\begin{aligned}
\dot{\xi}_{1} & =K_{1} \varphi\left(\xi_{1}, z-G \xi_{1}\right)-D \cdot \xi_{1}+\widetilde{F}_{1}, \\
\dot{z} & =-D \cdot z+G \cdot \widetilde{F}_{1}+\widetilde{F}_{2} .
\end{aligned}
$$

The main achievement of the change of coordinates is that the dynamics of the auxiliary state variables is independent of the reaction kinetics. The asymptotic observer equations are derived as follows (from (36) and (37)) [31]:

$$
\begin{aligned}
& {\left[\begin{array}{l}
\dot{z}_{\text {lest }} \\
\dot{z}_{2 \text { est }} \\
\dot{z}_{3 \text { est }}
\end{array}\right]=-D\left[\begin{array}{c}
z_{\text {lest }} \\
z_{2 \text { est }} \\
z_{3 \text { est }}
\end{array}\right]+\left[\begin{array}{cc}
\frac{-1}{k_{1}} & \frac{1}{k_{3}} \\
\frac{k_{5}}{k_{1}} & 0 \\
\frac{k_{6}}{k_{1}} & \frac{-k_{7}}{k_{3}}
\end{array}\right]\left[\begin{array}{l}
F_{1} \\
F_{3}
\end{array}\right]+\left[\begin{array}{c}
F_{2} \\
0 \\
0
\end{array}\right],} \\
& {\left[\begin{array}{l}
\dot{S}_{\text {2est }} \\
\dot{P}_{\text {2est }} \\
\dot{P}_{3 \text { est }}
\end{array}\right]=\left[\begin{array}{c}
z_{\text {lest }} \\
z_{\text {2est }} \\
z_{3 \text { est }}
\end{array}\right]-\left[\begin{array}{cc}
\frac{-1}{k_{1}} & \frac{1}{k_{3}} \\
\frac{k_{5}}{k_{1}} & 0 \\
\frac{k_{6}}{k_{1}} & \frac{-k_{7}}{k_{3}}
\end{array}\right]\left[\begin{array}{l}
S_{1} \\
P_{1}
\end{array}\right] .}
\end{aligned}
$$

The asymptotic observer can be used for the implementation of the adaptive law (35).

\section{Results and Discussion}

The behavior and the performance of the proposed estimation and control algorithms were analyzed by using intensive 


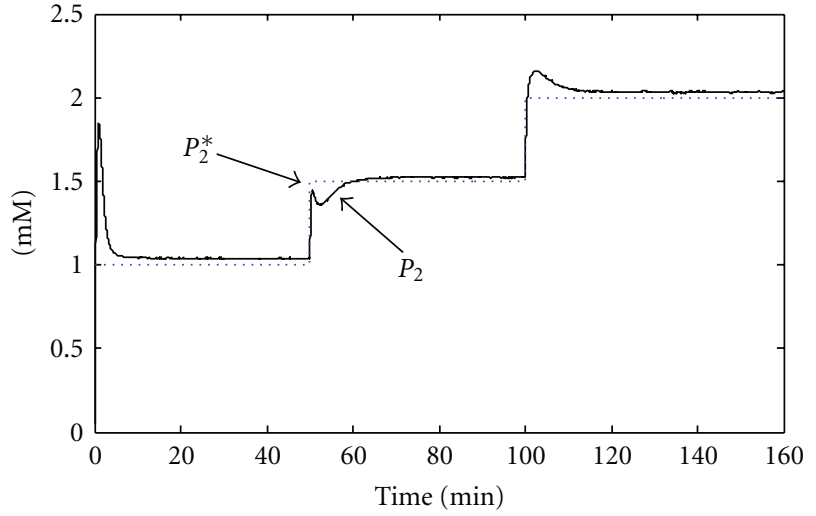

(a)

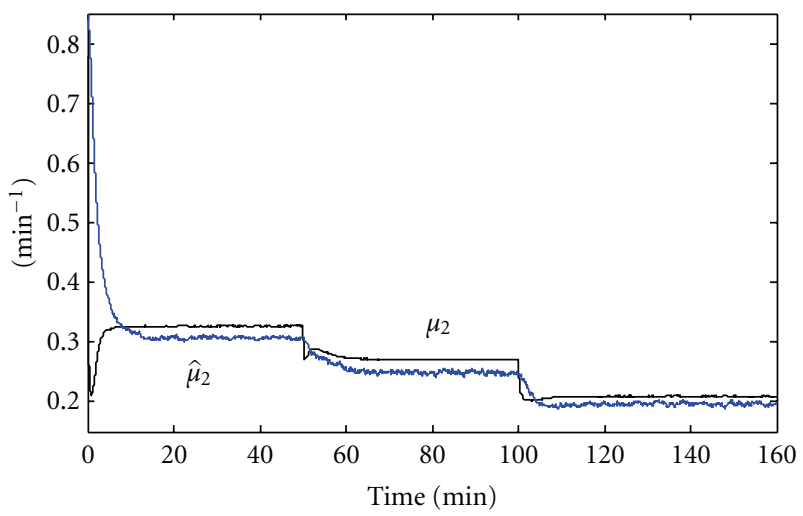

(c)

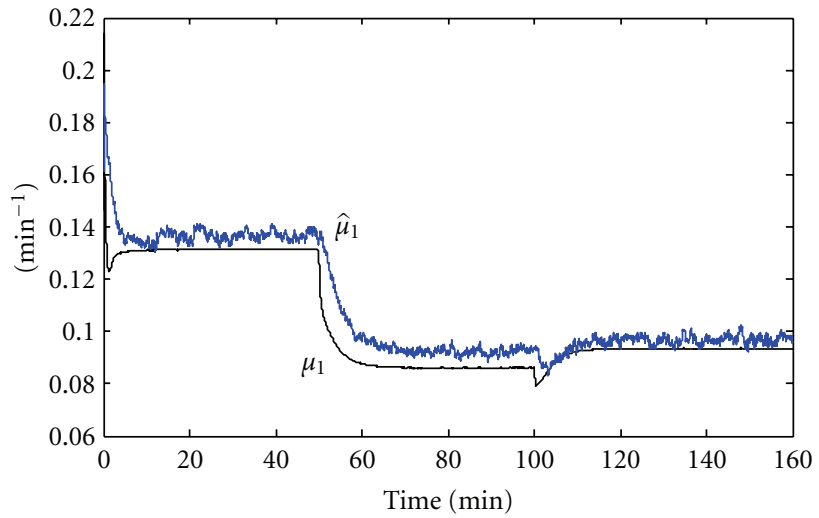

(b)

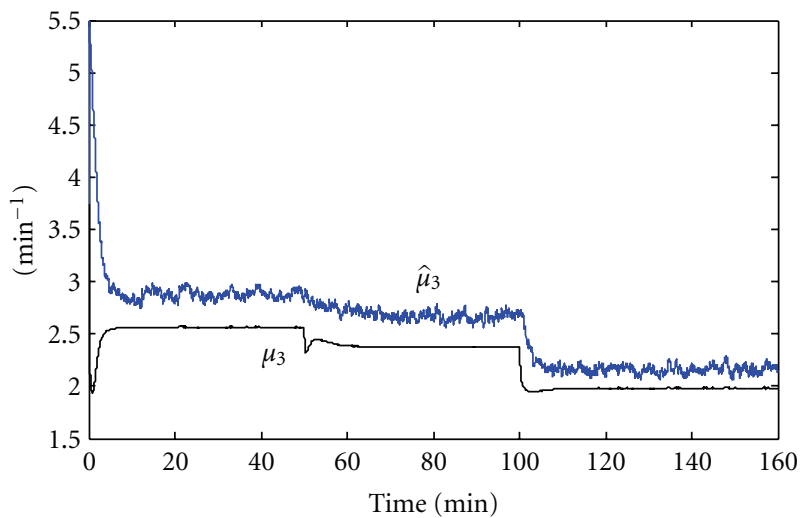

(d)

FIgURE 6: Simulation results-adaptive control law (noisy measurements). Panel (a) shows the profile of the output $y=P_{2}$ (ampicillin concentration) versus the reference $y^{*}=P_{2}^{*}$, in the adaptive case, when noisy measurements of $S_{1}$ and $S_{2}$ are used in both estimation and control algorithms. Panels (b), (c), and (d) present the profiles of specific reaction rates $\mu_{1}, \mu_{2}$, and $\mu_{3}$ together with their estimates $\hat{\mu}_{1}, \hat{\mu}_{2}$ and $\hat{\mu}_{3}$, respectively, in the case of noisy measurements.

TABLE 1: Bioprocess parameters values.

\begin{tabular}{lc}
\hline Parameter & Value \\
\hline$k_{\text {cat1 }}$ & $0.546(\mathrm{mM} / \mathrm{UI} \min )$ \\
$k_{\text {cat2 }}$ & $1.857(\mathrm{mM} / \mathrm{UI} \min )$ \\
$C_{\mathrm{EZ}}$ & $30(\mathrm{UI} / \mathrm{mlgel})$ \\
$k_{\mathrm{EN}}$ & $22.57(\mathrm{mM})$ \\
$k_{\mathrm{AE}}$ & $1.05(\mathrm{mM})$ \\
$k_{\mathrm{EA}}$ & $32.13(\mathrm{mM})$ \\
$K_{m 1}$ & $15.32(\mathrm{mM})$ \\
$K_{m 2}$ & $12.83(\mathrm{mM})$ \\
$F_{1}$ & $2(\mathrm{mM} / \mathrm{min})$ \\
$F_{2}$ & $2.5(\mathrm{mM} / \mathrm{min})$ \\
$F_{3}$ & $1(\mathrm{mM} / \mathrm{min})$ \\
$k_{1}$ & 0.7 \\
$k_{i}, i=2, \ldots, 7$ & 1 \\
\hline
\end{tabular}

simulations. The simulations were performed in MATLAB environment (registered trademark of The MathWorks Inc., USA). The fed-batch bioprocess has been simulated by numerical integration of the basic model equations (18),
(21)-(23). The values of kinetic parameters and of yield coefficients used in simulations are presented in Table 1 $[23,31]$.

Three simulation scenarios were taken into consideration.

(i) The exact linearizing control law (32) was implemented for the bioprocess (18), with the design parameter $\lambda=3$. The closed loop system was tested for a step profile of the ampicillin concentration reference. This simulation case is a kind of benchmark, because all the parameters and the reaction rates are considered to be known (and given by the relations (21)-(23)). A parametric disturbance was considered in the feed rate $F_{2}$, which has a $20 \%$ decrease of its nominal value (between $t=50 \mathrm{~min}$ and $t=100 \mathrm{~min}$ ). The simulation results are presented in Figure 4. Panel (a) shows the time profiles of $\xi_{1}=S_{1}, \xi_{2}=S_{2}, \xi_{3}=P_{1}$, and $\xi_{5}=$ $P_{3}$ (i.e., 6-APA, PGME, PG, and methanol concentrations, resp.). In panel (b) the evolution of the specific reaction rates is depicted, while in panel (c) the output (ampicillin concentration) versus the reference profile is shown. Panel (d) presents the control action, that is, the evolution of the dilution rate. From these simulation results, it can be seen a very good behavior of the controlled bioprocess. 
TABLE 2: Performance criterion results (free-noise data).

\begin{tabular}{lccc}
\hline Tuning parameter & $I_{1}$ & $I_{2}$ & $I_{3}$ \\
\hline$\theta=1$ & 0.01029 & 0.03230 & 0.08333 \\
$\theta=2$ & 0.00321 & 0.01789 & 0.03768 \\
$\theta=5$ & 0.00225 & 0.00849 & 0.01124 \\
$\theta=50$ & 0.00039 & 0.00052 & 0.00126 \\
\hline
\end{tabular}

TABle 3: Performance criterion results (noisy measurements).

\begin{tabular}{lccc}
\hline Tuning parameter & $I_{1}$ & $I_{2}$ & $I_{3}$ \\
\hline$\theta=1$ & 0.06370 & 0.03392 & 0.16932 \\
$\theta=2$ & 0.09196 & 0.02198 & 0.35544 \\
$\theta=5$ & 0.62471 & 0.24404 & 1.59703 \\
$\theta=50$ & - (divergent) & - (divergent) & - (divergent) \\
\hline
\end{tabular}

(ii) In the second simulation scenario, an adaptive version of the control law was implemented in the same conditions as in previous case. This adaptive controller consists of the high gain observer (28) and the control law (33). Therefore, the specific growth rates $\mu_{1}, \mu_{2}$, and $\mu_{3}$ were considered to be unknown. The kinetic expressions (21)(23) were introduced only for simulation; so these models were not used in the process of observer design. The main goal of the estimator (28) is to reconstitute the time evolution of $\mu_{1}, \mu_{2}$, and $\mu_{3}$ from the measurements of $S_{1}, S_{2}, P_{1}$, and $P_{2}$ obtained from the simulation.

The value of the tuning parameter was set to $\theta=2$. The on-line estimations provided by the high gain observer (28) were used in the control law (33) - in fact only $\hat{\mu}_{1}$ and $\hat{\mu}_{3}$ are used here. The results in this simulation case are shown in Figure 5. Panel (a) presents the output versus the reference profile, and panels (b), (c), and (d) show the time evolution of the estimated specific reaction rates versus their "true" profiles. In this scenario, the obtained results are good, despite the uncertainty in the bioprocess kinetics.

(iii) In order to test the robustness of the proposed estimation and control algorithms to noisy measurements, the behavior of the controlled bioprocess was also analyzed for noisy data of $S_{1}$ and $S_{2}$. The measurements of these concentrations are considered to be vitiated by an additive Gaussian noise, with zero mean and amplitude equal to 5\% of the free-noise values. The same version of the adaptive control law (28), (33) was used in this case. The obtained results are presented in Figure 6. As in previous case, panel (a) shows the output versus the reference profile, and panels (b), (c), and (d) depict the time evolution of the estimated specific reaction rates versus their "true" profiles.

It can be seen that the behavior of the controlled process is quite good, in spite of the combined action of noisy measurements, parametric disturbance, and kinetics uncertainty. The specific reaction rates $\mu_{1}$ and $\mu_{3}$ seem to be a little bit more sensible to noise.

A lot of supplementary simulations were performed for the other versions of adaptive control laws, such as (29), (34) and (28), (35). In all situations, a good behavior of the closed loop system was obtained. The results illustrate that the highgain observer provides accurate estimates of the kinetic rates. It can be seen that the noisy measurement induces some noisy estimates of the kinetics, but the noise effect is limited (however, this effect can be reduced for lower values of the tuning parameter). Several comparisons and comments regarding the behavior and the performance of the on-line estimation strategy can be achieved. Some remarks can be done by visualization of estimation errors $\tilde{\mu}_{1}=\mu_{1}-\hat{\mu}_{1}, \tilde{\mu}_{2}=$ $\mu_{2}-\hat{\mu}_{2}$ and $\tilde{\mu}_{3}=\mu_{3}-\hat{\mu}_{3}$. However, accurate comparisons can be realized by considering a criterion, for example, one based on averaged square estimation errors [3]:

$$
\begin{aligned}
& I_{1}=\frac{1}{T_{S}} \int_{0}^{T_{S}} \tilde{\mu}_{1}^{2}(t) d t, \\
& I_{2}=\frac{1}{T_{S}} \int_{0}^{T_{S}} \tilde{\mu}_{2}^{2}(t) d t, \\
& I_{3}=\frac{1}{T_{S}} \int_{0}^{T_{S}} \tilde{\mu}_{3}^{2}(t) d t,
\end{aligned}
$$

where $T_{S}$ is the total simulation time.

The values of $I_{1}, I_{2}$, and $I_{3}$ computed for different values of tuning parameter and free noise data are given in Table 2, and for vitiated measurements in Table 3. It results that the precision can be increased if the tuning parameter is bigger. The problem for a large value of $\theta$ is that the observer becomes noise sensitive. Notice that the estimation error can be made as small as wished if we choose greater values of $\theta$. The problem for a large value of $\theta$ is that the observer becomes noise sensitive. Therefore, the value of the tuning parameter is a compromise between a good estimation and the noise rejection. The obtained results concerning the noise sensitivity of the high gain observers are similar with those discussed in several works, such as [35-38].

\section{Conclusions}

The bond graph modeling approach constitutes a noteworthy option to the classical modeling in the case of complex bioprocesses. The ampicillin synthesis process was modeled in a natural way via pseudo bond graphs, and the obtained model was used for estimators and controllers design. One of the key advantages of the bond graph modeling of bioprocesses is the possibility to reuse the models, for example, in the interconnected bioreactors. The application of this feature is beyond of the scope of the present paper, but it is of crucial importance in other bioprocesses. However, it should be mentioned that the obtained model is only validated for the specific enzyme preparation used (but the proposed method can be utilized with adequate modifications when the enzyme is changed).

To overcome problems such as the kinetics uncertainties and the lack of on-line measurements, a high gain observer was designed and implemented for the on-line reconstruction of the specific reaction rates. The advantages of this kind of estimator are the simplicity of design, the good convergence and stability properties, and the accuracy of estimates (especially for free noise data). Another 
important advantage is the fact that the tuning of one single design parameter is necessary. The estimation results can be improved if the tuning parameter is chosen higher in value, but only if the measurements are free noise. Contrarily, the observer becomes noise sensitive and it is possible that the estimates of kinetics cannot be utilized in the control law design.

The simulation results show a good behavior of the adaptive controlled ampicillin synthesis bioprocess. The proposed adaptive control law was obtained by combining an exact linearizing control law with the kinetics estimator. The control goal, that is, the preservation of a high ampicillin concentration, is achieved despite the action of disturbances and noisy measurements. The bond graph modeling estimation and control strategies can be also applied to other processes belonging to the nonlinear class of bioprocesses considered in the present study.

\section{Acknowledgment}

This work was supported by CNCSIS UEFISCDI, Romania, Projects PNII-IDEI 548/2008 and PNII-RU 108/2010.

\section{References}

[1] B. Bastin and D. Dochain, On-Line Estimation and Adaptive Control of Bioreactors, Elsevier, Amsterdam, The Netherlands, 1990.

[2] K. Schügerl, "Progress in monitoring, modeling and control of bioprocesses during the last 20 years," Journal of Biotechnology, vol. 85, no. 2, pp. 149-173, 2001.

[3] D. Selişteanu, M. Roman, and D. Şendrescu, "Pseudo Bond Graph modelling and on-line estimation of unknown kinetics for a wastewater biodegradation process," Simulation Modelling Practice and Theory, vol. 18, no. 9, pp. 1297-1313, 2010.

[4] D. Karnopp and R. Rosenberg, System Dynamics: A Unified Approach, John Wiley, New York, NY, USA, 1974.

[5] W. Borutzky, Bond Graph Methodology. Development and Analysis of Multidisciplinary Dynamic System Models, Springer, London, UK, 2009.

[6] J. L. Baliño, "Galerkin finite element method for incompressible thermofluid flows framed within the bond graph theory," Simulation Modelling Practice and Theory, vol. 17, no. 1, pp. 35-49, 2009.

[7] F. Couenne, C. Jallut, B. Maschke, P. C. Breedveld, and M. Tayakout, "Bond graph modelling for chemical reactors," Mathematical and Computer Modelling of Dynamical Systems, vol. 12, no. 2-3, pp. 159-174, 2006.

[8] C. Heny, D. Simanca, and M. Delgado, "Pseudo-bond graph model and simulation of a continuous stirred tank reactor," Journal of the Franklin Institute, vol. 337, no. 1, pp. 21-42, 2000.

[9] V. Díaz-Zuccarini, D. Rafirou, J. LeFevre, D. R. Hose, and P. V. Lawford, "Systemic modelling and computational physiology: the application of Bond Graph boundary conditions for 3D cardiovascular models," Simulation Modelling Practice and Theory, vol. 17, no. 1, pp. 125-136, 2009.

[10] X. Zhang, K. A. Hoo, and D. Overland, "Bond Graph modeling of an integrated biological wastewater treatment system," in Proceedings of the American Institute of Chemical Engineers Annual Meeting, p. 479e, San Francisco, Calif, USA, 2006.
[11] M. Roman and D. Selişteanu, "Pseudo bond graph modelling of wastewater treatment bioprocesses," SIMULATIONTransactions of the Society of Modeling and Simulation International, vol. 88, no. 2, pp. 233-251, 2012.

[12] C. Pichardo-Almarza, A. Rahmani, G. Dauphin-Tanguy, and M. Delgado, "Proportional-integral observer for systems modelled by bond graphs," Simulation Modelling Practice and Theory, vol. 13, no. 3, pp. 179-211, 2005.

[13] M. Farza, K. Busawon, and H. Hammouri, "Simple nonlinear observers for on-line estimation of kinetic rates in bioreactors," Automatica, vol. 34, no. 3, pp. 301-318, 1998.

[14] J. P. Gauthier, H. Hammouri, and S. Othman, "A simple observer for nonlinear systems applications to bioreactors," IEEE Transactions on Automatic Control, vol. 37, no. 6, pp. 875-880, 1992.

[15] N. P. Guerra, P. Fajardo, C. Fuciños et al., "Modelling the biphasic growth and product formation by enterococcus faecium CECT 410 in realkalized fed-batch fermentations in whey," Journal of Biomedicine and Biotechnology, vol. 2010, Article ID 290286, 16 pages, 2010.

[16] D. Şendrescu, E. Petre, and E. Bobaşu, "Structural identifiability of some biotechnological systems," in Proceedings of The WSEAS International Conference on Environment, Ecosystems and Development (EED '07), pp. 49-55, Puerto de la Cruz, Spain, 2007.

[17] H. K. Khalil, "High-gain observers in nonlinear feedback control," in Proceedings of the International Conference on Control, Automation and Systems, pp. 47-57, Seoul, Republic of Korea, 2008.

[18] D. Selişteanu, E. Petre, and V. B. Rǎsvan, "Sliding mode and adaptive sliding-mode control of a class of nonlinear bioprocesses," International Journal of Adaptive Control and Signal Processing, vol. 21, no. 8-9, pp. 795-822, 2007.

[19] D. Şendrescu, "Nonlinear model predictive control of a depollution bioprocess," in Proceedings of 3rd Pacific-Asia Conference on Circuits, Communications and System (PACCS '11), vol. 1, pp. 1-4, Wuhan, China, 2011.

[20] D. Selișteanu, E. Petre, M. Roman, D. Popescu, and E. Bobașu, "On-line estimation of unknown kinetics for the enzymatic synthesis of ampicillin," in Proceedings of the International Carpathian Control Conference, pp. 335-340, Velke Karlovice, Czech Republic, 2011.

[21] J. P. Hou and J. W. Poole, "The amino acid nature of ampicillin and related penicillins," Journal of Pharmaceutical Sciences, vol. 58, no. 12, pp. 1510-1515, 1969.

[22] A. L. O. Ferreira, L. R. B. Gonçalves, R. C. Giordano, and R. L. C. Giordano, "A simplified kinetic model for the side reactions occurring during the enzymatic synthesis of ampicillin," Brazilian Journal of Chemical Engineering, vol. 17, no. 4 , pp. 835-839, 2000.

[23] L. R. B. Gonçalves, R. Fernandez-Lafuente, J. M. Guisán, and R. L. C. Giordano, "A kinetic study of synthesis of amoxicillin using penicillin G acylase immobilized on agarose," Applied Biochemistry and Biotechnology, vol. 84-86, pp. 931-945, 2000.

[24] S. A. W. Jager, P. A. Jekel, and D. B. Janssen, "Hybrid penicillin acylases with improved properties for synthesis of $\beta$-lactam antibiotics," Enzyme and Microbial Technology, vol. 40, no. 5, pp. 1335-1344, 2007.

[25] J. M. Guisan, "Immobilization of enzymes as the 21st Century begins: an already solved problem or still an exciting challenge?" in Immobilization of Enzymes and Cells, J. M. Guisan, Ed., vol. 22 of Methods in Biotechnology, pp. 1-13, 2006.

[26] K. Buchholz, V. Kasche, and U. T. Theo Bornscheuer, Biocatalysts and Enzyme Technology, Wiley-VCH, Weinheim, Germany, 2005. 
[27] M. Terreni, J. G. Tchamkam, U. Sarnataro, S. Rocchietti, R. Fernández-Lafuente, and J. M. Guisán, "Influence of substrate structure on PGA-catalyzed acylations. Evaluation of different approaches for the enzymatic synthesis of cefonicid," Advanced Synthesis and Catalysis, vol. 347, no. 1, pp. 121-128, 2005.

[28] C. Mateo, J. M. Palomo, G. Fernandez-Lorente, J. M. Guisan, and R. Fernandez-Lafuente, "Improvement of enzyme activity, stability and selectivity via immobilization techniques," Enzyme and Microbial Technology, vol. 40, no. 6, pp. 1451$1463,2007$.

[29] K. Hernandez and R. Fernandez-Lafuente, "Control of protein immobilization: coupling immobilization and site-directed mutagenesis to improve biocatalyst or biosensor performance," Enzyme and Microbial Technology, vol. 48, no. 2, pp. 107-122, 2011.

[30] G. Volpato, R. C. Rodrigues, and R. Fernandez-Lafuente, "Use of enzymes in the production of semi-synthetic penicillins and cephalosporins: drawbacks and perspectives," Current Medicinal Chemistry, vol. 17, no. 32, pp. 3855-3873, 2010.

[31] E. C. Ferreira, Identification and adaptive control of biotechnological processes, Ph.D. thesis, University of Porto, 1995.

[32] A. Isidori, Nonlinear Control Systems, Springer, London, UK, 3rd edition, 1995.

[33] A. K. P. B. Siqueira, L. R. B. Gonçalves, R. C. Giordano, R. L. C. Giordano, and A. L. O. Ferreira, "Ampicillin synthesis catalyzed by penicillin acylase: effect of $\mathrm{pH}$ and type of carriers on selectivity and yield," in Proceedings of 2nd Mercosur Congress on Chemical Engineering, Brazil, 2005.

[34] M. P. De Arruda Ribeiro and R. D. C. Giordano, "Variational calculus (optimal control) applied to the optimization of the enzymatic synthesis of ampicillin," Brazilian Archives of Biology and Technology, vol. 48, pp. 19-28, 2005.

[35] J. H. Ahrens and H. K. Khalil, "High-gain observers in the presence of measurement noise: a switched-gain approach," Automatica, vol. 45, no. 4, pp. 936-943, 2009.

[36] V. Andrieu, L. Praly, and A. Astolfi, "High gain observers with updated gain and homogeneous correction terms," Automatica, vol. 45, no. 2, pp. 422-428, 2009.

[37] N. Boizot, E. Busvelle, and J. P. Gauthier, "An adaptive highgain observer for nonlinear systems," Automatica, vol. 46, no. 9, pp. 1483-1488, 2010.

[38] M. Farza, M. Oueder, R. Benabdennour, and M. M'Saad, "High gain observer with updated gain for a class of MIMO systems," International Journal of Control, vol. 84, no. 2, pp. 270-280, 2011. 

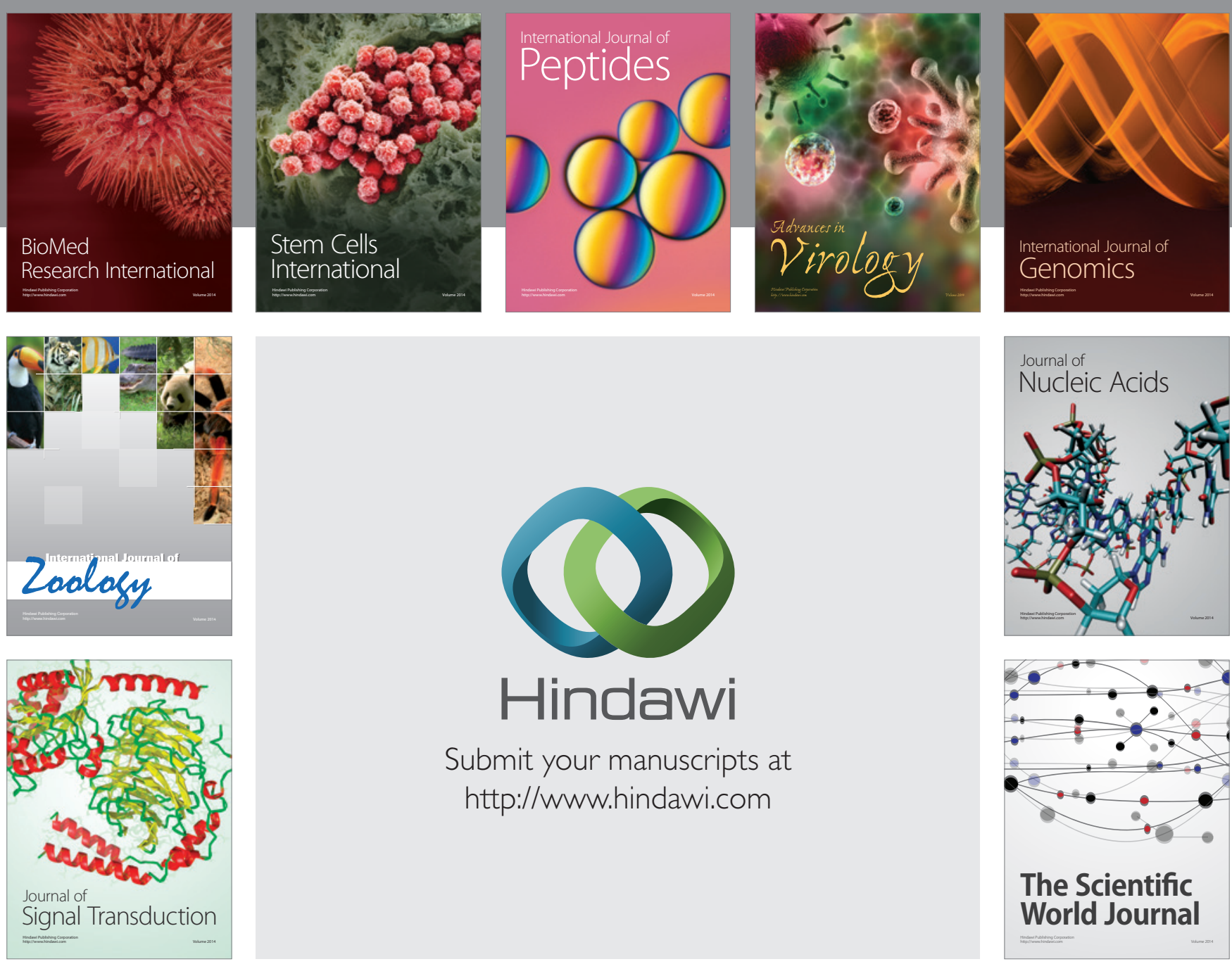

Submit your manuscripts at

http://www.hindawi.com
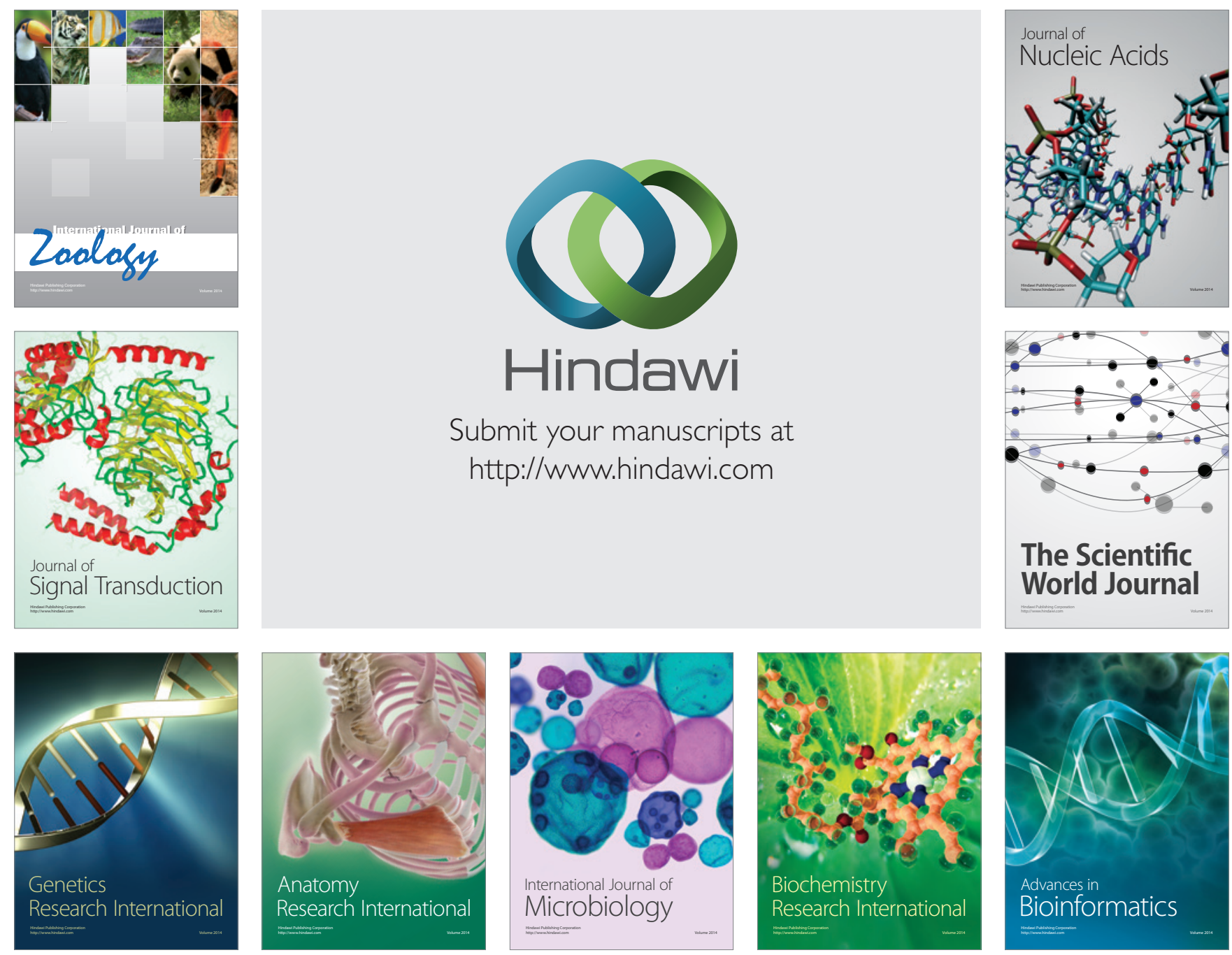

The Scientific World Journal
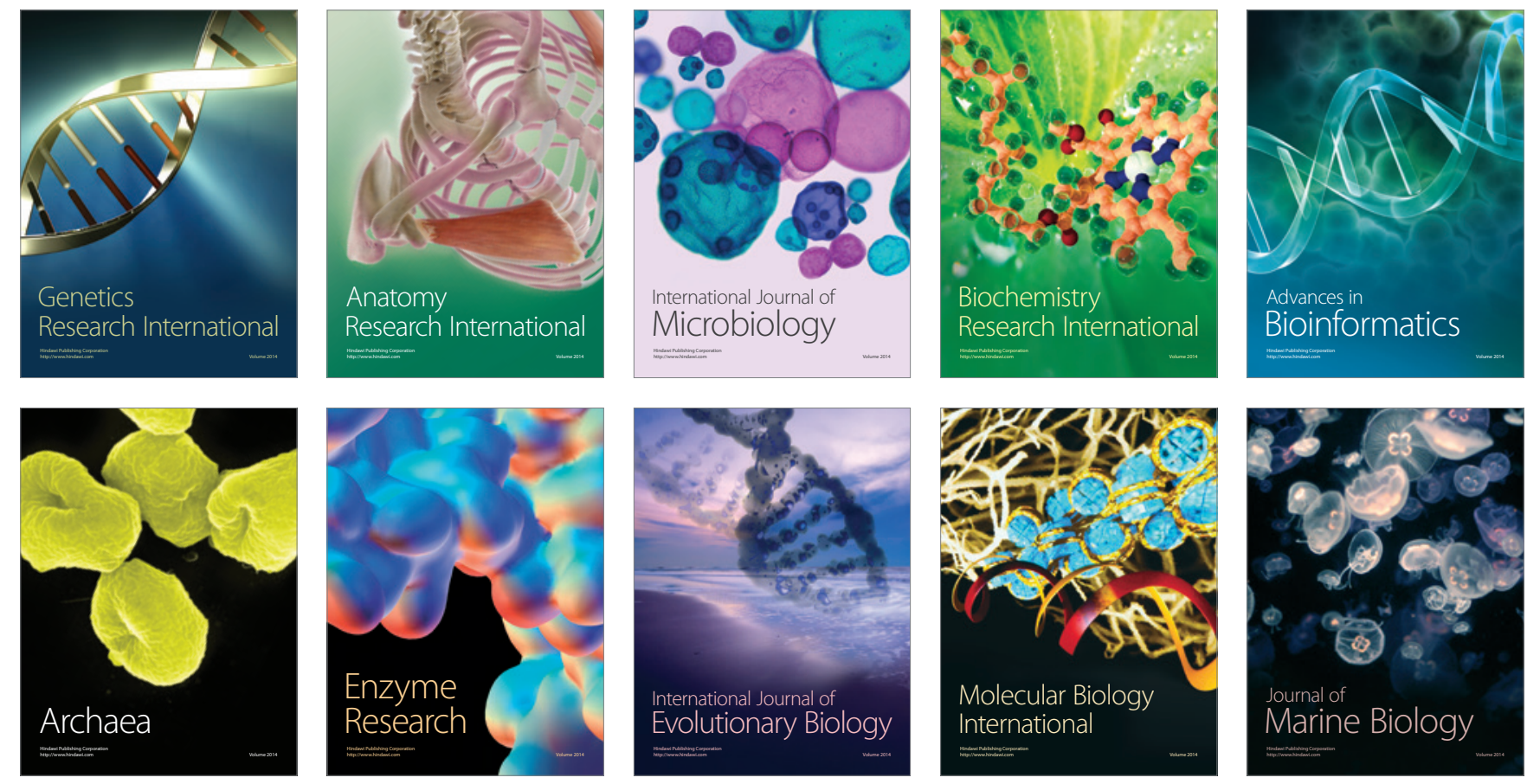